This item was submitted to Loughborough's Research Repository by the author.

Items in Figshare are protected by copyright, with all rights reserved, unless otherwise indicated.

\title{
Marginal values and returns to scale for nonparametric production frontiers
}

PLEASE CITE THE PUBLISHED VERSION

http://dx.doi.org/10.1287/opre.2015.1457

\section{PUBLISHER}

(c) INFORMS (Institute for Operations Research and Management Sciences)

\section{VERSION}

AM (Accepted Manuscript)

\section{PUBLISHER STATEMENT}

This work is made available according to the conditions of the Creative Commons Attribution-NonCommercialNoDerivatives 4.0 International (CC BY-NC-ND 4.0) licence. Full details of this licence are available at: https://creativecommons.org/licenses/by-nc-nd/4.0/

\section{LICENCE}

CC BY-NC-ND 4.0

\section{REPOSITORY RECORD}

Podinovski, Victor, Robert G. Chambers, Kazim Baris Atici, and Iryna D. Deineko. 2016. "Marginal Values and Returns to Scale for Nonparametric Production Frontiers". Loughborough University. https://hdl.handle.net/2134/19950. 


\title{
Submitted to Operations Research \\ manuscript (Please, provide the mansucript number!)
}

\section{Marginal Values and Returns to Scale for Nonparametric Production Frontiers}

\author{
Victor V. Podinovski \\ School of Business and Economics, Loughborough University, Loughborough LE11 3TU, United Kingdom, \\ v.podinovski@lboro.ac.uk \\ Robert G. Chambers \\ Department of Agricultural and Resource Economics, University of Maryland, College Park, Maryland 20742, \\ rchamber@umd.edu \\ Kazim Baris Atici \\ Department of Business Administration, Hacettepe University, Ankara 06800, Turkey, kba@hacettepe.edu.tr \\ Iryna D. Deineko \\ Warwick Business School, University of Warwick, Coventry CV4 7AL, UK, iryna_deineko@btinternet.com
}

\begin{abstract}
We present a unifying linear programming approach to the calculation of various directional derivatives for a very large class of production frontiers of data envelopment analysis (DEA). Special cases of this include different marginal rates, the scale elasticity and a spectrum of partial and mixed elasticity measures. Our development applies to any polyhedral production technology including, to name a few, the conventional variable and constant returns-to-scale DEA technologies, their extensions with weight restrictions, technologies with weakly disposable undesirable outputs and network DEA models. Furthermore, our development provides a general method for the characterization of returns to scale (RTS) in any polyhedral technology. The new approach effectively removes the need to develop bespoke models for the RTS characterization and calculation of marginal rates and elasticity measures for each particular technology.
\end{abstract}

Key words: data envelopment analysis; elasticity measures; returns to scale; marginal rates; weight restrictions; undesirable outputs

\section{Introduction}

Data envelopment analysis (DEA) is an established nonparametric methodology of efficiency analysis (Cooper et al. 2007, Thanassoulis et al. 2008). While the main purpose of DEA is the assessment of efficiency of organizational units, its methodology can be used to address a number of other tasks important for decision and policy making. One of these is the analysis of productive potential of units that are already operating efficiently, in response to particular changes to their input and output profiles. The standard indicators for this type of analysis are various marginal rates (e.g., the rate of substitution between two inputs) and elasticity measures (e.g., the scale elasticity). Closely related to the latter is the characterization of returns to scale (RTS) of efficient units - see, 
e.g., Banker et al. (2011) and Zelenyuk (2013) for a review. The link between the types of RTS and scale elasticity is highlighted by Førsund and Hjalmarsson (2004) who point out that the latter is a quantitative measure of the strength of the RTS characterization.

The growing literature on the topics of marginal characteristics and RTS is typically limited to the conventional constant and variable returns-to-scale (CRS and VRS) technologies. Because the production frontiers of these technologies are not explicitly known and are not smooth, the analysis of their marginal rates and elasticities is not straightforward. As noted by many researchers (Charnes et al. 1985, Banker at Maindiratta 1986, Olesen and Petersen 1996, Krivonozhko et al. 2004), earlier results on marginal rates and scale elasticity were obtained only for relative interior points of efficient facets of the technology and were not rigorously proved at the extreme points corresponding to observed efficient units.

In more recent studies the above difficulties were overcome by the introduction of directional, or one-sided, differential characteristics (Chambers and Färe 2008). Hadjicostas and Soteriou (2006) and Podinovski et al. (2009) obtained linear programs suitable for the calculation of the left-hand and right-hand scale elasticity over the entire frontier of the VRS technology, including its extreme points. A more general approach was developed by Podinovski and Førsund (2010) and Atici and Podinovski (2012) who defined a large class of one-sided partial elasticity measures in the VRS and CRS technologies and the corresponding RTS classification.

Despite the recent advances, the existing methodologies for the analysis of differential characteristics of efficient frontiers are still incomplete in several respects.

- The existing methods for the calculation of marginal rates and elasticity measures, including the approaches of Podinovski and Førsund (2010) and Atici and Podinovski (2012), apply only to the standard VRS and CRS DEA technologies. It is not clear how similar rates and measures could be computed in other technologies. An example of this is the VRS and CRS technologies with production trade-offs or mathematically equivalent to them weight restrictions (Podinovski 2004b, Podinovski and Bouzdine-Chameeva 2013). ${ }^{1}$ Another example is the VRS technology with weakly disposable undesirable outputs (Kuosmanen 2005).

- Several studies suggest bespoke computational methods suitable for the RTS characterization of efficient units in specific technologies. Examples include technologies with weight restrictions (Tone 2001, Korhonen et al. 2011) and network DEA technologies (Sahoo et al. 2014). These methods are not designed for the calculation of the underlying scale elasticity. It is also unclear how the notion of RTS introduced for such technologies relates to the corresponding scale elasticity, which remains undefined.

- In a general setting we may be interested in the marginal rate of response (or elasticity of response) of a subvector of inputs or outputs to another subvector, where both subvectors are 
Podinovski et al.: Marginal Values for Production Frontiers

changed either proportionally or in particular directions. For example, this may involve the response of all outputs to marginal changes of discretionary inputs, keeping exogenous environmental inputs fixed (Ruggiero 2000). Balk et al. (2015) provide theoretical results for the calculation of such marginal rates for smooth production frontiers defined by a known production function. For nonsmooth DEA frontiers whose function representation is unknown, there exist no suitable calculation methodods, even in the standard VRS and CRS technologies.

In our paper we overcome the above limitations by developing a general theoretical and computational approach that applies to a very large class of production technologies and yields various marginal rates and elasticity measures. This approach extends the earlier results of Podinovski and Førsund (2010) which become special cases of the new development. Our new results are based on two generalizing observations.

First, we note that the conventional VRS and CRS technologies are examples of a large class of technologies that are polyhedral sets. We refer to such technologies as polyhedral technologies. This class also includes technologies with production trade-offs and weight restrictions, their variants with negative inputs and outputs, some technologies with weakly disposable undesirable outputs and network DEA technologies. Unlike the existing methods that rely on specific statements of technology (e.g., VRS or CRS), our approach does not depend on this and relies only on the general description of technology as a polyhedral set.

Second, we note that various marginal rates and elasticity measures can be obtained as directional derivatives of an appropriately specified directional distance function of Chambers et al. (1998), taken in an appropriately chosen direction.

The main contribution of our paper is the development of a unified linear programming approach to the calculation of directional marginal values (rates and elasticities) in any polyhedral technology. In particular, this approach can be used for the calculation of scale elasticity and the corresponding RTS characterization, in all commonly used polyhedral technologies for which no suitable methods currently exist. This general approach effectively removes the need to develop bespoke methods of computation for each type of marginal rate or elasticity (and RTS characterization), for each particular technology, except for the cases where specialist methods offer computational advantages (Krivonozhko et al. 2014).

To illustrate our development, we consider three numerical examples. These involve the computation of different marginal values in two common polyhedral technologies for which no programming methods are available. Although these examples are different, we show that all computations can be performed by solving the same universal linear programs developed in our paper, that only need an appropriate and straightforward specification. These programs can be used with any other polyhedral technology in a similar fashion. 


\section{A Theorem of Directional Marginal Values}

Below we present our main theoretical result in the general form. Its special cases are explored in subsequent sections.

\subsection{Polyhedral Technologies}

Let $I$ and $O$ be the sets of inputs and outputs, and let $|I|=m>0$ and $|O|=s>0$. A production unit (member of technology) is represented by the pair $(X, Y)$, where $X \in \mathbb{R}^{m}$ and $Y \in \mathbb{R}^{s}$ are the vectors of its inputs and outputs, respectively.

For simplicity, our main development concerns technologies that satisfy the assumption of free disposability of inputs and outputs. The general case of any polyhedral technology requires a minor modification of our results and is considered in $\S 2.5$ below.

We define a (freely disposable) technology $\mathcal{T}$ as the set of all units $(X, Y)$ for which there exist a vector $\hat{\lambda} \in \mathbb{R}^{q}$ and vectors of input and output slacks $S_{X} \in \mathbb{R}^{m}$ and $S_{Y} \in \mathbb{R}^{s}$, respectively, such that the following conditions are true:

$$
\begin{aligned}
& \hat{X} \hat{\lambda}+S_{X}=X, \\
& \hat{Y} \hat{\lambda}-S_{Y}=Y, \\
& \hat{U} \hat{\lambda}=U_{o} \\
& \hat{\lambda}, S_{X}, S_{Y} \geq 0 .
\end{aligned}
$$

In the above formulation, $\hat{X}$ and $\hat{Y}$ are, respectively, the input and output data matrices of dimensions $m \times q$ and $s \times q$. These matrices may consist of the columns representing the inputs and outputs of the observed units, and may also incorporate additional data, as discussed in examples below. The vector equality (1.3) is optional and specifies any additional conditions on the vector $\hat{\lambda}$, for example, the normalizing equality in the VRS model. In this equality, $U_{o} \in \mathbb{R}^{p}$ is a constant vector and $\hat{U}$ is a matrix of dimension $p \times q .^{2}$

The definition of technology $\mathcal{T}$ typically requires that some or all inputs and outputs of units in $\mathcal{T}$ are nonnegative. To simplify notation, we assume that all inputs and outputs are nonnegative: ${ }^{3}$

$$
X \geq 0, \quad Y \geq 0
$$

Proposition 1. Technology $\mathcal{T}$ defined by conditions (1) and (2) is a polyhedral (and therefore convex) set.

Two straightforward examples of technologies stated in the form (1) and (2) are the conventional CRS and VRS technologies (Charnes et al. 1978, Banker et al. 1984). For both technologies the 
Podinovski et al.: Marginal Values for Production Frontiers

columns of matrices $\hat{X}$ and $\hat{Y}$ are, respectively, the input and output vectors $X_{j}$ and $Y_{j}$ of the observed units $j=1, \ldots, q$. In the case of CRS, condition (1.3) is omitted and, in the case of VRS, it includes the normalizing equality $1^{\top} \hat{\lambda}=1$. Another common example is the VRS and CRS technologies that incorporate weight restrictions or production trade-offs - this is considered in $\S 6$.

Further examples include the non-increasing and non-decreasing RTS technologies (Färe and Grosskopf 1985, Seiford and Thrall 1990), the hybrid returns-to-scale (HRS) technology (Podinovski 2004a, Podinovski et al. 2014) and its cone extension (Podinovski 2009), technologies exhibiting multiple variable proportionality (Cook and Zhu 2011), and the VRS and CRS technologies with negative data (Emrouznejad et al. 2010). Network DEA models are also based on technologies in which the sets of vectors of inputs and final outputs comprise a polyhedral technology stated in the form (1) and (2)—see, e.g., Sahoo et al. (2014).

\subsection{Directional Response Functions}

In a slight generalization of the framework of Podinovski and Førsund (2010), consider the partition of all inputs and outputs into three mutually disjoint sets $A, B$ and $C$, where the first two sets are not empty. Then any unit $\left(X_{o}, Y_{o}\right) \in \mathcal{T}$ can be stated in the following general form:

$$
\left(X_{o}, Y_{o}\right)=\left(X_{o}^{A}, X_{o}^{B}, X_{o}^{C}, Y_{o}^{A}, Y_{o}^{B}, Y_{o}^{C}\right)
$$

Suppose we have chosen two vectors, $\Delta^{A}=\left(\Delta_{X}^{A}, \Delta_{Y}^{A}\right)$ and $\Delta^{B}=\left(\Delta_{X}^{B}, \Delta_{Y}^{B}\right)$, that describe the directions of perturbation to the inputs and outputs in the sets $A$ and $B$, respectively. (For example, in this notation, the subvector $\Delta_{X}^{A}$ corresponds to the inputs included in the set $A$. This subvector is undefined if no inputs are included in $A$.)

Let unit $\left(X_{o}, Y_{o}\right)$ be located on the boundary of technology $\mathcal{T}$. (A more precise requirement is stated below in Assumption 1.) Our goal is to investigate the marginal change of the inputs and outputs of the unit $\left(X_{o}, Y_{o}\right)$ included in the set $B$ in the direction $\Delta^{B}$, in response to a marginal change of its inputs and outputs in the set $A$ in the direction $\Delta^{A}$, while keeping the measures in $C$ constant, provided the resulting perturbed unit remains on the boundary of technology $\mathcal{T}$.

The following definition and assumption are central to our analysis. In these statements, the amounts of change of inputs and outputs in the direction of vectors $\Delta^{A}$ and $\Delta^{B}$ are described by the scalars $\gamma$ and $\varphi$, respectively.

Definition 1. For the unit $\left(X_{o}, Y_{o}\right) \in \mathcal{T}$ and vectors $\Delta^{A}$ and $\Delta^{B}$, the directional response function $\bar{\varphi}(\gamma)$ is the optimal value (provided it exists - see Proposition 2 below)

$$
\bar{\varphi}(\gamma)=\max \left\{\varphi \mid\left(X_{o}^{A}+\gamma \Delta_{X}^{A}, X_{o}^{B}+\varphi \Delta_{X}^{B}, X_{o}^{C}, Y_{o}^{A}+\gamma \Delta_{Y}^{A}, Y_{o}^{B}+\varphi \Delta_{Y}^{B}, Y_{o}^{C}\right) \in \mathcal{T}, \varphi \in \mathbb{R}\right\}
$$


Assumption 1. The unit $\left(X_{o}, Y_{o}\right)$ is weakly efficient in the direction $\Delta^{B}$ :

$$
\bar{\varphi}(0)=\max \left\{\varphi \mid\left(X_{o}^{A}, X_{o}^{B}+\varphi \Delta_{X}^{B}, X_{o}^{C}, Y_{o}^{A}, Y_{o}^{B}+\varphi \Delta_{Y}^{B}, Y_{o}^{C}\right) \in \mathcal{T}, \varphi \in \mathbb{R}\right\}=0 .
$$

Note that $\bar{\varphi}(0)$ is the directional distance function of Chambers et al. (1998) evaluated at the point $\left(X_{o}, Y_{o}\right)$ in the direction of vector $\Delta^{B}$. The function $\bar{\varphi}(\gamma)$ is the same directional distance function evaluated at the perturbed point $\left(X_{o}^{A}+\gamma \Delta_{X}^{A}, X_{o}^{B}, X_{o}^{C}, Y_{o}^{A}+\gamma \Delta_{Y}^{A}, Y_{o}^{B}, Y_{o}^{C}\right)$. Therefore, the directional response function $\bar{\varphi}(\gamma)$ is the directional distance function parameterized by scalar $\gamma$.

Assumption 1 means that the inputs and outputs of the unit $\left(X_{o}, Y_{o}\right)$ included in the set $B$ cannot be changed in the direction $\Delta^{B}$ within technology $\mathcal{T}$, while the remaining inputs and outputs are kept unchanged. In other words, the unit $\left(X_{o}, Y_{o}\right)$ is (weakly) efficient in the direction $\Delta^{B}$ and is, therefore, a boundary point of $\mathcal{T} .^{5}$

To see the meaning of the directional response function $\bar{\varphi}(\gamma)$, suppose the input and output components $X_{o}^{A}$ and $Y_{o}^{A}$ of the unit $\left(X_{o}, Y_{o}\right)$ are changed by the amounts $\gamma \Delta_{X}^{A}$ and $\gamma \Delta_{Y}^{A}$, respectively. Then the maximum amount of change to components $X_{o}^{B}$ and $Y_{o}^{B}$ possible in the technology and performed in the direction of vectors $\Delta_{X}^{B}$ and $\Delta_{Y}^{B}$ is equal to $\bar{\varphi}(\gamma) \Delta_{X}^{B}$ and $\bar{\varphi}(\gamma) \Delta_{Y}^{B}$, respectively.

The following two examples further illustrate the above definition and assumption.

EXAmPle 1. Suppose the set $B$ includes all outputs and no inputs. If we define $\Delta_{Y}^{B}=Y_{o}$, then formula (4) maximizes the full vector of outputs without changing the inputs. Therefore, Assumption 1 means that the unit $\left(X_{o}, Y_{o}\right)$ is output radial efficient in $\mathcal{T}$. Similarly, let the set $B$ include all inputs and no outputs, and let $\Delta_{X}^{B}=-X_{o}$. Then Assumption 1 implies that the unit $\left(X_{o}, Y_{o}\right)$ is input radial efficient. Note, however, that if in the latter setting $\Delta_{X}^{B}=X_{o}$, the maximum in (4) is unbounded and Assumption 1 is not satisfied.

ExAmple 2. Suppose the set $A$ consists of a single input $i^{*}$ and the set $B$ consists of a single output $r^{*}$, while all the other inputs and outputs are included in the set $C$. Let the only components of the single-dimension vectors $\Delta_{X}^{A}$ and $\Delta_{Y}^{B}$ be equal to 1 . According to Assumption 1 , the unit $\left(X_{o}, Y_{o}\right)$ produces the maximum amount of output $r^{*}$ : the latter cannot be increased if the other inputs and outputs do not change. In this scenario the directional response function is the maximum amount $\bar{\varphi}(\gamma)$ of output $r^{*}$ that can additionally be produced in the technology provided the single input $i^{*}$ is increased by $\gamma$ units.

Taking into account the definition of technology $\mathcal{T}$ by conditions (1) and (2), the function $\bar{\varphi}(\gamma)$ is obtained as the optimal value in the following linear program:

$$
\begin{array}{ll}
\bar{\varphi}(\gamma)=\max & \varphi \\
\text { subject to } & \hat{X}^{A} \hat{\lambda}+S_{X}^{A}=X_{o}^{A}+\gamma \Delta_{X}^{A},
\end{array}
$$




$$
\begin{aligned}
& \hat{X}^{B} \hat{\lambda}+S_{X}^{B}=X_{o}^{B}+\varphi \Delta_{X}^{B}, \\
& \hat{X}^{C} \hat{\lambda}+S_{X}^{C}=X_{o}^{C}, \\
& \hat{Y}^{A} \hat{\lambda}-S_{Y}^{A}=Y_{o}^{A}+\gamma \Delta_{Y}^{A}, \\
& \hat{Y}^{B} \hat{\lambda}-S_{Y}^{B}=Y_{o}^{B}+\varphi \Delta_{Y}^{B}, \\
& \hat{Y}^{C} \hat{\lambda}-S_{Y}^{C}=Y_{o}^{C} \\
& \hat{U} \hat{\lambda}=U_{o} \\
& D_{X}^{A}=X_{o}^{A}+\gamma \Delta_{X}^{A}, \\
& D_{Y}^{A}=Y_{o}^{A}+\gamma \Delta_{Y}^{A}, \\
& D_{X}^{B}=X_{o}^{B}+\varphi \Delta_{X}^{B}, \\
& D_{Y}^{B}=Y_{o}^{B}+\varphi \Delta_{Y}^{B}, \\
& \hat{\lambda}, S_{X}, S_{Y}, D_{X}^{A}, D_{Y}^{A}, D_{X}^{B}, D_{Y}^{B} \geq 0, \varphi \text { sign free. }
\end{aligned}
$$

In this program, the superscripts $A, B$ and $C$ denote the groups of rows of matrices $\hat{X}$ and $\hat{Y}$, and components of vectors $X_{o}, Y_{o}, S_{X}$ and $S_{Y}$ that correspond to the sets $A, B$ and $C$, respectively. Equalities (5.9)-(5.12) incorporate variable vectors $D_{X}^{A}, D_{Y}^{A}, D_{X}^{B}$ and $D_{Y}^{B}$ and represent nonnegativity conditions (2).

Note that (5) is a linear program in which variable $\varphi$ is maximized for each value of $\gamma$ (the latter is a fixed parameter not used for the optimization). Let $\Gamma$ be the domain of the function $\bar{\varphi}(\gamma)$. This is the set of all values $\gamma$ for which program (5) is feasible and has a finite optimal value $\bar{\varphi}(\gamma)$. By Assumption 1 , the value $\gamma=0$ belongs to the domain $\Gamma$, and the latter is not an empty set.

Using well-known results of sensitivity analysis in linear programming (see, e.g., Roos et al. 2005), we have the following properties of the function $\bar{\varphi}(\gamma)$ and its domain $\Gamma$.

Proposition 2. (i) $\Gamma$ is a closed interval.

(ii) $\bar{\varphi}(\gamma)$ is a continuous, concave and piecewise linear function on its domain $\Gamma$.

Note that, depending on the specification of technology $\mathcal{T}$, choice of the unit $\left(X_{o}, Y_{o}\right)$ and directions $\Delta^{A}$ and $\Delta^{B}$, the interval $\Gamma$ may, in some cases, be a single point or an unbounded set.

\subsection{The Main Theorem}

As follows from Proposition 2, the function $\bar{\varphi}(\gamma)$ has a finite right-hand derivative at any $\gamma$ that is not the right extreme point of $\Gamma$. Similarly, its left-hand derivative exists and is finite at any $\gamma$ that is not the left extreme point of $\Gamma$. It is also clear that the two one-sided derivatives are generally different. 
Of the most interest to us are the one-sided derivatives of the function $\bar{\varphi}(\gamma)$ at $\gamma=0$. As clarified in $\S \S 3$ and 4 , the choice of different sets $A$ and $B$, and directions $\Delta^{A}$ and $\Delta^{B}$, leads to the definition of different marginal characteristics at the unit $\left(X_{o}, Y_{o}\right)$. These include, among others, the standard marginal rates and elasticity measures, in particular, the scale elasticity. ${ }^{6}$

It is now clear that the notion of one-sided derivatives of the function $\bar{\varphi}(\gamma)$ at $\gamma=0$ is central to the definition of a spectrum of marginal characteristics of production frontiers. Our task is to develop programming methods for their calculation. This is achieved by the statement of our main result below. Its proof relies on the known theorem of marginal values in linear programming (Mills 1952) and its more recent variant presented in Roos et al. (2005).

In the formulation of the following main theorem, $\nu=\left(\nu^{A}, \nu^{B}, \nu^{C}\right)$ is the dual vector to constraints (5.2)-(5.4), and $\mu=\left(\mu^{A}, \mu^{B}, \mu^{C}\right)$ is the negated dual vector to constraints (5.5)-(5.7). ${ }^{7}$ The vectors $\omega, \rho, \sigma, \xi$ and $\zeta$ are dual to constraints (5.8)-(5.12), respectively. Furthermore, the unit $\left(X_{o}, Y_{o}\right) \in \mathcal{T}$ may be either observed or unobserved.

Theorem 1. Consider any unit $\left(X_{o}, Y_{o}\right) \in \mathcal{T}$.

1. If Assumption 1 is satisfied then the following four statements are true:

(i) If $\gamma=0$ is not the right extreme point of domain $\Gamma$, then the right-hand derivative $\bar{\varphi}_{+}^{\prime}(0)$ exists, is finite and is equal to the optimal value of the linear program

$$
\begin{aligned}
\bar{\varphi}_{+}^{\prime}(0)=\min & \left(\Delta_{X}^{A}\right)^{\top} \nu^{A}-\left(\Delta_{Y}^{A}\right)^{\top} \mu^{A}+\left(\Delta_{X}^{A}\right)^{\top} \rho+\left(\Delta_{Y}^{A}\right)^{\top} \sigma \\
\text { subject to } & -\left(\Delta_{X}^{B}\right)^{\top} \nu^{B}+\left(\Delta_{Y}^{B}\right)^{\top} \mu^{B}-\left(\Delta_{X}^{B}\right)^{\top} \xi-\left(\Delta_{Y}^{B}\right)^{\top} \zeta=1, \\
& X_{o}^{\top} \nu-Y_{o}^{\top} \mu+U_{o}^{\top} \omega+\left(X_{o}^{A}\right)^{\top} \rho+\left(Y_{o}^{A}\right)^{\top} \sigma+\left(X_{o}^{B}\right)^{\top} \xi+\left(Y_{o}^{B}\right)^{\top} \zeta=0, \\
& \hat{X}^{\top} \nu-\hat{Y}^{\top} \mu+\hat{U}^{\top} \omega \geq 0, \\
& \mu, \nu, \rho, \sigma, \xi, \zeta \geq 0, \omega \text { sign free vector. }
\end{aligned}
$$

(ii) If $\gamma=0$ is the right extreme point of $\Gamma$, then the optimal value of program (6) is unbounded.

(iii) If $\gamma=0$ is not the left extreme point of $\Gamma$, then the left-hand derivative $\bar{\varphi}_{+}^{\prime}(0)$ exists, is finite and

$$
\begin{aligned}
\bar{\varphi}_{-}^{\prime}(0)=\max & \left(\Delta_{X}^{A}\right)^{\top} \nu^{A}-\left(\Delta_{Y}^{A}\right)^{\top} \mu^{A}+\left(\Delta_{X}^{A}\right)^{\top} \rho+\left(\Delta_{Y}^{A}\right)^{\top} \sigma \\
\text { subject to } & (6.2)-(6.5) .
\end{aligned}
$$

(iv) If $\gamma=0$ is the left extreme point of $\Gamma$, then the optimal value of program (7) is unbounded. 2. If Assumption 1 is not satisfied, the programs (6) and (7) are infeasible.

Comparing programs (6) and (7), we obtain the following statement. (Note that it also follows from the statement (ii) of Proposition 2.)

Proposition 3. If $\gamma=0$ is an interior point of $\Gamma$ then $\bar{\varphi}_{-}^{\prime}(0) \geq \bar{\varphi}_{+}^{\prime}(0)$. 
Theorem 1 is stated assuming the nonnegativity condition (2) on all inputs and outputs. In a more general case only some, or none, of the inputs and outputs may be required to be nonnegative, and programs (6) and (7) are simplified. To be specific, suppose that none of the inputs and outputs in the sets $A$ and $B$ are required to be nonnegative. Then the nonnegativity conditions (5.9)-(5.12) are omitted from program (5). Consequently, the dual vectors $\rho, \sigma, \xi$ and $\zeta$, and associated terms in programs (6) and (7) are removed.

\subsection{Simplifying Conditions}

Suppose that all components of vectors $X_{o}^{A}, Y_{o}^{A}, X_{o}^{B}$ and $Y_{o}^{B}$ are strictly positive. It is intuitively clear that in this case the nonnegativity conditions (2) do not affect the definition of the function $\bar{\varphi}(\gamma)$ in a sufficiently small neighborhood of $\gamma=0$. Consequently, conditions (2) can be ignored in the calculation of the one-sided derivatives $\bar{\varphi}_{+}^{\prime}(0)$ and $\bar{\varphi}_{-}^{\prime}(0)$.

In particular, program (6) can be restated in the following simplified form

$$
\begin{aligned}
\bar{\varphi}_{+}^{\prime}(0)=\min & \left(\Delta_{X}^{A}\right)^{\top} \nu^{A}-\left(\Delta_{Y}^{A}\right)^{\top} \mu^{A} \\
\text { subject to } & -\left(\Delta_{X}^{B}\right)^{\top} \nu^{B}+\left(\Delta_{Y}^{B}\right)^{\top} \mu^{B}=1, \\
& X_{o}^{\top} \nu-Y_{o}^{\top} \mu+U_{o}^{\top} \omega=0, \\
& \hat{X}^{\top} \nu-\hat{Y}^{\top} \mu+\hat{U}^{\top} \omega \geq 0, \\
& \mu, \nu \geq 0, \omega \text { sign free vector. }
\end{aligned}
$$

Similarly, program (7) becomes

$$
\begin{array}{cl}
\bar{\varphi}_{-}^{\prime}(0)=\max & \left(\Delta_{X}^{A}\right)^{\top} \nu^{A}-\left(\Delta_{Y}^{A}\right)^{\top} \mu^{A} \\
\text { subject to } & (8.2)-(8.5) .
\end{array}
$$

Proposition 4. Let all components of vectors $X_{o}^{A}, Y_{o}^{A}, X_{o}^{B}$ and $Y_{o}^{B}$ be strictly positive. Then Theorem 1 is true if programs (6) and (7) are substituted by programs (8) and (9), respectively.

\subsection{General Polyhedral Technologies}

Any technology stated in the form (1) and (2) satisfies the assumption of free disposability for all inputs and outputs. Some polyhedral technologies do not satisfy this property and cannot, therefore, be expressed by these conditions. Examples include the CRS technology that exhibits weak disposability with respect to undesirable outputs (see Färe and Grosskopf 2004, Chen and Delmas 2012), and its VRS analogue (Kuosmanen 2005).

If some input $i^{*}$ or output $r^{*}$ does not satisfy the assumption of free disposability, the corresponding slack component $\left(S_{X}\right)_{i^{*}}$ or $\left(S_{Y}\right)_{r^{*}}$ is removed from conditions (1.1) and (1.2). In this case 
Theorem 1 remains true, but the corresponding dual variables $\nu_{i^{*}}$ and $\mu_{r^{*}}$ in programs (6) and (7) become sign-free variables. We illustrate this in the example in $\S 6.3$.

This last observation means that Theorem 1 applies to any polyhedral technology stated in terms of units $(X, Y) .^{8,9}$ All Propositions 1-4 also remain true without any modification.

\section{Elasticity Measures}

Below we use Theorem 1 to extend the notions of scale elasticity and other elasticity measures introduced by Podinovski and Førsund (2010) and Atici and Podinovski (2012) in the VRS and CRS technologies, to the entire class of polyhedral technologies. This naturally leads to the the corresponding RTS characterization of units in such technologies.

\subsection{Definition of Elasticity Measures}

In order to simplify our development, we assume that technology $\mathcal{T}$ is defined by conditions (1) and (2). Our results further extend to any general polyhedral technology taking into account the minor modifications discussed in $\S 2.5$.

Following Podinovski and Førsund (2010), we give the following definition.

Definition 2. For the unit $\left(X_{o}, Y_{o}\right) \in \mathcal{T}$ and the sets $A, B$ and $C$, the proportional response function $\bar{\beta}(\alpha)$ is the following optimal value (provided it exists):

$$
\bar{\beta}(\alpha)=\max \left\{\beta \mid\left(\alpha X_{o}^{A}, \beta X_{o}^{B}, X_{o}^{C}, \alpha Y_{o}^{A}, \beta Y_{o}^{B}, Y_{o}^{C}\right) \in \mathcal{T}, \beta \in \mathbb{R}\right\} .
$$

The function $\bar{\beta}(\alpha)$ represents the maximum proportion of vectors $X_{o}^{B}$ and $Y_{o}^{B}$ feasible in technology $\mathcal{T}$, if the vectors $X_{o}^{A}$ and $Y_{o}^{A}$ change in proportion $\alpha$, and the vectors $X_{o}^{C}$ and $Y_{o}^{C}$ are kept constant. ${ }^{10}$ We further require that the unit $\left(X_{o}, Y_{o}\right)$ be (weakly) efficient in the direction defined by the vectors $X_{o}^{B}$ and $Y_{o}^{B}$ :

Assumption 2. $\bar{\beta}(1)=1$.

Assuming the required derivatives exist, define the one-sided elasticities of response of the pair of vectors $\left(X_{o}^{B}, Y_{o}^{B}\right)$ with respect to proportional changes of the pair of vectors $\left(X_{o}^{A}, Y_{o}^{A}\right)$ as follows:

$$
\varepsilon_{A, B}^{+}\left(X_{o}, Y_{o}\right)=\bar{\beta}_{+}^{\prime}(1), \quad \varepsilon_{A, B}^{-}\left(X_{o}, Y_{o}\right)=\bar{\beta}_{-}^{\prime}(1) .
$$

The meaning of the one-sided elasticities (11) is straightforward. Let the pair of vectors $\left(X_{o}^{A}, Y_{o}^{A}\right)$ change in some proportion $\alpha=1+\gamma$, where $\gamma$ is marginally small. Then, if $\gamma>0$, the maximum proportion of the pair of vectors $\left(X_{o}^{B}, Y_{o}^{B}\right)$ possible in technology $\mathcal{T}$ is equal to $1+\varepsilon_{A, B}^{+}\left(X_{o}, Y_{o}\right) \times \gamma$. In simple words, a proportional increase of vectors $X_{o}^{A}$ and $Y_{o}^{A}$ by, say, $1 \%$ (corresponding to $\gamma=0.01)$ causes the responding vectors $X_{o}^{B}$ and $Y_{o}^{B}$ to change by $\varepsilon_{A, B}^{+}\left(X_{o}, Y_{o}\right)$ percent. (Note that $\varepsilon_{A, B}^{+}\left(X_{o}, Y_{o}\right)$ may generally be positive, negative or zero.) Similarly, if $\gamma<0$, the maximum proportion of the pair of vectors $\left(X_{o}^{B}, Y_{o}^{B}\right)$ possible in $\mathcal{T}$ is $1+\varepsilon_{A, B}^{-}\left(X_{o}, Y_{o}\right) \times \gamma$. 
Podinovski et al.: Marginal Values for Production Frontiers

\subsection{Calculation of Elasticity Measures}

Below we show that the one-sided elasticities $\varepsilon_{A, B}^{+}\left(X_{o}, Y_{o}\right)$ and $\varepsilon_{A, B}^{-}\left(X_{o}, Y_{o}\right)$ can be calculated using the main Theorem 1. Following Chambers et al. (1998), we first note that the proportional response function $\bar{\beta}(\alpha)$ can be viewed as a special case of the directional response function $\bar{\varphi}(\gamma)$. Indeed, consider the function $\bar{\varphi}(\gamma)$ defined by formula $(3)$ in which

$$
\Delta_{X}^{A}=X_{o}^{A}, \quad \Delta_{Y}^{A}=Y_{o}^{A}, \quad \Delta_{X}^{B}=X_{o}^{B}, \quad \Delta_{Y}^{B}=Y_{o}^{B}
$$

Then

$$
\bar{\varphi}(\gamma)=\max \left\{\varphi \mid\left(X_{o}^{A}+\gamma X_{o}^{A}, X_{o}^{B}+\varphi X_{o}^{B}, X_{o}^{C}, Y_{o}^{A}+\gamma Y_{o}^{A}, Y_{o}^{B}+\varphi Y_{o}^{B}, Y_{o}^{C}\right) \in \mathcal{T}, \varphi \in \mathbb{R}\right\}
$$

Denote $\alpha=1+\gamma$ and $\beta=1+\varphi$. Comparing (13) with (10), we have $\bar{\beta}(\alpha)=1+\bar{\varphi}(\gamma)$, where $\gamma=\alpha-1$. Therefore, taking into account (11) and assuming the required derivatives exist, we have

$$
\begin{aligned}
& \varepsilon_{A, B}^{+}\left(X_{o}, Y_{o}\right)=\bar{\beta}_{+}^{\prime}(1)=\bar{\varphi}_{+}^{\prime}(0), \\
& \varepsilon_{A, B}^{-}\left(X_{o}, Y_{o}\right)=\bar{\beta}_{-}^{\prime}(1)=\bar{\varphi}_{-}^{\prime}(0) .
\end{aligned}
$$

Equalities (14) imply that $\varepsilon_{A, B}^{+}\left(X_{o}, Y_{o}\right)$ and $\varepsilon_{A, B}^{-}\left(X_{o}, Y_{o}\right)$ can be computed using Theorem 1 . In fact, programs (6) and (7) of Theorem 1 can be simplified. Indeed, it is clear that a marginal proportional change of vectors $X_{o}^{A}, Y_{o}^{A}, X_{o}^{B}$ and $Y_{o}^{B}$ by $\alpha$ and $\beta$ close to 1 in formula (10) cannot violate the nonnegativity conditions (2) (if specified), and the latter should be redundant for the calculation of elasticity measures. This in turn means that vectors $\rho, \sigma, \xi$ and $\zeta$ in programs (6) and (7) are also redundant and may be omitted. The simplified program (6) takes on the form

$$
\begin{aligned}
\varepsilon_{A, B}^{+}\left(X_{o}, Y_{o}\right)=\bar{\varphi}_{+}^{\prime}(0)=\min & \left(X_{o}^{A}\right)^{\top} \nu^{A}-\left(Y_{o}^{A}\right)^{\top} \mu^{A} \\
\text { subject to } & -\left(X_{o}^{B}\right)^{\top} \nu^{B}+\left(Y_{o}^{B}\right)^{\top} \mu^{B}=1, \\
\text { and } & (8.3)-(8.5) .
\end{aligned}
$$

Similarly, program (7) takes on the form

$$
\begin{aligned}
\varepsilon_{A, B}^{-}\left(X_{o}, Y_{o}\right)=\bar{\varphi}_{-}^{\prime}(0)=\max & \left(X_{o}^{A}\right)^{\top} \nu^{A}-\left(Y_{o}^{A}\right)^{\top} \mu^{A} \\
\text { subject to } & -\left(X_{o}^{B}\right)^{\top} \nu^{B}+\left(Y_{o}^{B}\right)^{\top} \mu^{B}=1, \\
\text { and } & (8.3)-(8.5) .
\end{aligned}
$$

Proposition 5. Let equalities (12) be true. Then Theorem 1 remains true if programs (6) and (7) are substituted by programs (15) and (16), respectively. ${ }^{11}$

Taking into account programs (15) and (16), and assuming that both one-sided elasticities exist ${ }^{12}$, we obtain the following analogue of Proposition 3:

$$
\varepsilon_{A, B}^{-}\left(X_{o}, Y_{o}\right) \geq \varepsilon_{A, B}^{+}\left(X_{o}, Y_{o}\right)
$$




\subsection{Scale Elasticity}

In the case of scale elasticity, $A=I$ is the set of all inputs and $B=O$ is the set of all outputs. Restating (15) with $X_{o}^{A}=X_{o}$ and $Y_{o}^{B}=Y_{o}$, the right-hand scale elasticity is found as

$$
\begin{aligned}
\varepsilon_{I, O}^{+}\left(X_{o}, Y_{o}\right)=\min & X_{o}^{\top} \nu \\
\text { subject to } & Y_{o}^{\top} \mu=1, \\
& X_{o}^{\top} \nu-Y_{o}^{\top} \mu+U_{o}^{\top} \omega=0, \\
& \hat{X}^{\top} \nu-\hat{Y}^{\top} \mu+\hat{U}^{\top} \omega \geq 0, \\
& \mu, \nu \geq 0, \omega \text { sign free vector. }
\end{aligned}
$$

Similarly, to calculate the left-hand scale elasticity, we solve

$$
\begin{aligned}
\varepsilon_{I, O}^{-}\left(X_{o}, Y_{o}\right)=\max & X_{o}^{\top} \nu \\
\text { subject to } & (18.2)-(18.5) .
\end{aligned}
$$

It is straightforward to show that the feasible regions of programs (18) and (19) coincide with the set $\Omega$ of optimal solutions $\langle\mu, \nu, \omega\rangle$ to the multiplier model that assesses the output radial efficiency of the unit $\left(X_{o}, Y_{o}\right)$ in technology $\mathcal{T}$ defined by conditions (1) and (2). ${ }^{13}$ Furthermore, as follows from equalities (18.2) and (18.3), the objective function of programs (18) and (19) can be restated as $X_{o}^{\top} \nu=1-U_{o}^{\top} \omega$. This allows us to rewrite both programs as follows:

$$
\begin{aligned}
& \varepsilon_{I, O}^{+}\left(X_{o}, Y_{o}\right)=1-\max _{\langle\mu, \nu, \omega\rangle \in \Omega}\left\{U_{o}^{\top} \omega\right\}, \\
& \varepsilon_{I, O}^{-}\left(X_{o}, Y_{o}\right)=1-\min _{\langle\mu, \nu, \omega\rangle \in \Omega}\left\{U_{o}^{\top} \omega\right\} .
\end{aligned}
$$

Programs (20) generalize the known formulae for one-sided scale elasticities in the conventional VRS technology:

$$
\begin{gathered}
\varepsilon_{I, O}^{+}\left(X_{o}, Y_{o}\right)=1-\omega^{\max }, \\
\varepsilon_{I, O}^{-}\left(X_{o}, Y_{o}\right)=1-\omega^{\min },
\end{gathered}
$$

where $\omega^{\max }$ and $\omega^{\min }$ are, respectively, the maximum and minimum optimal values of the variable $\omega$ dual to the normalizing equality $1^{\top} \lambda=1$ of the output-oriented VRS model. The identification of $\omega^{\max }$ and $\omega^{\text {min }}$ requires solving two linear programs - see, e.g., Fukuyama (2000) and Zelenyuk (2013). Obviously, formulae (21) are a special case of linear programs (20), and both are computationally equivalent to programs (18) and (19).

A similar expression of the one-sided elasticities $\varepsilon_{I, O}^{+}\left(X_{o}, Y_{o}\right)$ and $\varepsilon_{I, O}^{-}\left(X_{o}, Y_{o}\right)$ can be obtained via the set of optimal dual vectors $\omega$ in the input-oriented multiplier model. ${ }^{14}$ 
Podinovski et al.: Marginal Values for Production Frontiers

Article submitted to Operations Research; manuscript no. (Please, provide the mansucript number!)

\subsection{Returns to Scale}

Following Banker (1984) and Banker and Thrall (1992), in the DEA literature the RTS type of an efficient unit $\left(X_{o}, Y_{o}\right)$ operating in the VRS technology is conventionally determined by its one-sided scale elasticities as follows. ${ }^{15}$

Definition 3. The output radial efficient unit $\left(X_{o}, Y_{o}\right) \in \mathcal{T}_{\text {VRS }}$ exhibits:

(a) increasing returns to scale (IRS) if $1<\varepsilon_{I, O}^{+}\left(X_{o}, Y_{o}\right) \leq \varepsilon_{I, O}^{-}\left(X_{o}, Y_{o}\right)$,

(b) decreasing returns to scale (DRS) if $\varepsilon_{I, O}^{+}\left(X_{o}, Y_{o}\right) \leq \varepsilon_{I, O}^{-}\left(X_{o}, Y_{o}\right)<1$,

(c) constant returns to scale $(\mathrm{CRS})$ if $\varepsilon_{I, O}^{+}\left(X_{o}, Y_{o}\right) \leq 1 \leq \varepsilon_{I, O}^{-}\left(X_{o}, Y_{o}\right)$.

(In the above definition, we let $\varepsilon_{I, O}^{-}\left(X_{o}, Y_{o}\right)=+\infty$ if the optimal value of program (19) is unbounded. ${ }^{16}$ )

Using programs (18) and (19), we extend Definition 3 of the three types of RTS to any technology stated by conditions (1) and (2). We further extend this definition to any polyhedral technology by noting the comments made in $\S 2.5$. Furthermore, by taking different sets $A$ and $B$ in programs (15) and (16), we can define different types of partial RTS. Examples of this characterization and supporting computations are considered in $\S \S 6.1$ and 6.2.

It is worth noting that there is a growing interest in the DEA literature to specialist methods for the determination of RTS in particular technologies (Tone 2001, Korhonen et al. 2011, Sahoo et al. 2014). In contrast, our development based on programs (18) and (19) provides a universal method to the RTS characterization in any polyhedral technology. ${ }^{17}$

\section{Marginal Rates}

Below we use Theorem 1 for the calculation of one-sided marginal rates (partial derivatives) of a single input or output with respect to another input or output on the production frontier.

The novelty of this development is that the models presented below are universal for any polyhedral technology and allow us to perform required computations in technologies where no other methods are currently available. Furthermore, as discussed in $\S 5$, we do not need to check if the unit $\left(X_{o}, Y_{o}\right)$ at which the marginal rate is evaluated is weakly efficient in the sense of Assumption 1 , or if the marginal change in question is feasible in the technology - the required diagnostic is automatically obtained by the standard output of linear optimizers.

A computational example in $\S 6.3$ illustrates the theoretical development of this section.

\subsection{Marginal Product}

In economics, the marginal product is defined as the change in a single output $r^{*}$ associated with a marginal change in a single input $\tilde{i}$, holding all other outputs and inputs constant. In other words, 
the marginal product is the partial derivative of output $r^{*}$ with respect to input $\tilde{i}$ calculated on the production frontier and assuming the partial derivative exists. Because the frontiers of polyhedral technologies are generally not smooth, the required partial derivatives generally do not exist. This is naturally overcome by the introduction of one-sided marginal products that correspond to onesided partial derivatives. For example, the right-hand marginal product represents the change in an output associated with a marginally small positive change in an input holding all other inputs and outputs constant. The left-hand marginal product is defined similarly in the case of a marginally small negative change in the input.

To calculate the one-sided marginal products, let $A=\{\tilde{i}\}$ and $B=\left\{r^{*}\right\}$. We further define single-dimensional vectors $\Delta_{X}^{A}$ and $\Delta_{Y}^{B}$ such that $\left(\Delta_{X}^{A}\right)_{\tilde{i}}=1$ and $\left(\Delta_{Y}^{B}\right)_{r^{*}}=1$.

Consider any unit $\left(X_{o}, Y_{o}\right) \in \mathcal{T}$ that satisfies Assumption 1. This means that the unit $\left(X_{o}, Y_{o}\right)$ produces the maximum amount of output $r^{*}$ possible in technology $\mathcal{T}$, provided the remaining outputs and all inputs are kept constant. (A sufficient condition of this is that the unit $\left(X_{o}, Y_{o}\right)$ is Pareto-efficient, but this is not a necessary condition.)

As follows from Definition 1, the directional response function $\bar{\varphi}(\gamma)$ is equal to the maximum additional amount (number of units) $\varphi$ of output $r^{*}$ possible in the technology provided that input $\tilde{i}$ is changed by $\gamma$ units. Therefore, the right-hand and left-hand derivatives of the response function $\bar{\varphi}(\gamma)$ at $\gamma=0$ are equal to the right-hand and left-hand marginal products at the unit $\left(X_{o}, Y_{o}\right)$, respectively, provided the required derivatives exist. Thus, for example,

$$
\frac{\partial^{+} Y_{r^{*}}\left(X_{o}, Y_{o}\right)}{\partial X_{\tilde{i}}}=\lim _{\gamma \rightarrow 0+} \frac{\left[\left(Y_{o}\right)_{r^{*}}+\bar{\varphi}(\gamma)\right]-\left(Y_{o}\right)_{r^{*}}}{\left[\left(X_{o}\right)_{\tilde{i}}+\gamma\right]-\left(X_{o}\right)_{\tilde{i}}}=\lim _{\gamma \rightarrow 0+} \frac{\bar{\varphi}(\gamma)}{\gamma}=\bar{\varphi}_{+}^{\prime}(0)
$$

By Theorem 1, the one-sided marginal products can be found (or proved to be undefined) by solving programs (6) and (7), respectively, where the vectors $\Delta_{X}^{A}$ and $\Delta_{Y}^{B}$ are as stated above.

A particularly simple case arises if both the input $\tilde{i}$ and output $r^{*}$ of the unit $\left(X_{o}, Y_{o}\right)$ are strictly positive. This is a special case of the simplifying condition of Proposition 4. Restating program (8), the right-hand marginal product is calculated as

$$
\begin{aligned}
\frac{\partial^{+} Y_{r^{*}}\left(X_{o}, Y_{o}\right)}{\partial X_{\tilde{i}}}=\min & \nu_{\hat{i}} \\
\text { subject to } & \mu_{r^{*}}=1, \\
\text { and } & (8.3)-(8.5) .
\end{aligned}
$$

Similarly, the left-hand marginal product $\partial^{-} Y_{r^{*}}\left(X_{o}, Y_{o}\right) / \partial X_{\tilde{i}}$ is calculated by solving program (23) in which the minimization of objective function is replaced by its maximization. 
Podinovski et al.: Marginal Values for Production Frontiers

Article submitted to Operations Research; manuscript no. (Please, provide the mansucript number!)

\subsection{Marginal Rate of Transformation}

This is defined as the change of a single output $r^{*}$ required to maintain production feasibility and technical efficiency when another output $\tilde{r}$ is marginally changed. Similar to the case of marginal product, in a polyhedral technology we differentiate between the right-hand and left-hand marginal rates of transformation. These correspond to the right-hand and left-hand partial derivatives of output $r^{*}$ with respect to output $\tilde{r}$.

Let $A=\{\tilde{r}\}, B=\left\{r^{*}\right\},\left(\Delta_{Y}^{A}\right)_{\tilde{r}}=1$ and $\left(\Delta_{Y}^{B}\right)_{r^{*}}=1$. Using a similar derivation as in (22), it is straightforward to prove that the right-hand and left-hand marginal rates of transformation evaluated at the unit $\left(X_{o}, Y_{o}\right)$ are equal to the one-sided derivatives $\bar{\varphi}_{+}^{\prime}(0)$ and $\bar{\varphi}_{-}^{\prime}(0)$, respectively, and can be found by solving programs (6) and (7) of Theorem 1. If both outputs $\tilde{r}$ and $r^{*}$ of the unit $\left(X_{o}, Y_{o}\right)$ are strictly positive, these programs are replaced by simpler programs (8) and (9). For example, the former program takes on the form

$$
\begin{aligned}
\frac{\partial^{+} Y_{r^{*}}\left(X_{o}, Y_{o}\right)}{\partial Y_{\tilde{r}}}=\min & -\mu_{\tilde{r}} \\
\text { subject to } & \mu_{r^{*}}=1, \\
\text { and } & (8.3)-(8.5) .
\end{aligned}
$$

\subsection{Marginal Rate of Substitution}

This represents the change of a single input $i^{*}$ required to maintain production feasibility and technical efficiency when another single input $\tilde{i}$ marginally changes. In a polyhedral technology, the one-sided marginal rates of substitution are defined as the corresponding one-sided partial derivatives of input $i^{*}$ with respect to input $\tilde{i}$. Define the sets $A=\{\tilde{i}\}$ and $B=\left\{i^{*}\right\}$ and let $\left(\Delta_{X}^{A}\right)_{i}=1$. Note that in this case, in order to satisfy Assumption 1, we define the single component $\left(\Delta_{X}^{B}\right)_{i^{*}}=-1 .{ }^{18}$

In the described setting, the lowest level of input $i^{*}$ possible in the technology in response to the change of input $\tilde{i}$ by $\gamma$ units is equal to the difference $\left(X_{o}\right)_{i^{*}}-\tilde{\varphi}(\gamma)$. Then

$$
\frac{\partial^{+} X_{i^{*}}\left(X_{o}, Y_{o}\right)}{\partial X_{\tilde{i}}}=\lim _{\gamma \rightarrow 0+} \frac{\left[\left(X_{o}\right)_{i^{*}}-\bar{\varphi}(\gamma)\right]-\left(X_{o}\right)_{i^{*}}}{\left[\left(X_{o}\right)_{\tilde{i}}+\gamma\right]-\left(X_{o}\right)_{\tilde{i}}}=\lim _{\gamma \rightarrow 0+} \frac{-\bar{\varphi}(\gamma)}{\gamma}=-\bar{\varphi}_{+}^{\prime}(0) .
$$

Similarly, we have $\partial^{-} X_{i^{*}}\left(X_{o}, Y_{o}\right) / \partial X_{\tilde{i}}=-\bar{\varphi}_{-}^{\prime}(0)$. Therefore, both one-sided rates of substitution at the unit $\left(X_{o}, Y_{o}\right)$ can be calculated by negating the optimal values of properly stated programs (6) and (7), respectively. If both inputs $\tilde{i}$ and $i^{*}$ of the unit $\left(X_{o}, Y_{o}\right)$ are strictly positive, programs (6) and (7) are replaced by simpler programs (8) and (9). For example, negating the optimal value of the the former program, we have

$$
\begin{aligned}
\frac{\partial^{+} X_{i^{*}}\left(X_{o}, Y_{o}\right)}{\partial X_{\tilde{i}}}=-\min & \nu_{\tilde{i}} \quad\left(=\max -\nu_{\tilde{i}}\right) \\
\text { subject to } & \nu_{i^{*}}=1, \\
\text { and } & (8.3)-(8.5) .
\end{aligned}
$$


Finally note that programs (23)-(25), and their analogues for the left-hand derivatives apply to any technology $\mathcal{T}$ stated by conditions (1) and (2). In the standard VRS technology $\mathcal{T}_{\text {VRS }}$ these coincide with the known formulae (see, e.g., Rosen et al. 1998, Podinovski and Førsund 2010).

\section{Practical Considerations}

Below we discuss a procedure for practical use of Theorem 1. We first note that the input or output radial efficiency of the unit $\left(X_{o}, Y_{o}\right)$ is not required (and need not be checked) for the definition and calculation of directional marginal values, including elasticity measures. Instead, the use of Theorem 1 relies on Assumption 1 (which becomes Assumption 2 in the case of elasticity measures).

Furthermore, there is no need to check in advance whether either assumption is satisfied. For example, as follows from Theorem 1, Assumption 1 is satisfied if programs (6) and (7) (sharing the same feasible set) are feasible, and is not satisfied otherwise. Therefore, programs (6) and (7) can be solved over the entire set of observed units - the units that do not satisfy Assumptions 1 or 2 would simply make these two programs infeasible.

For linear programs (6) and (7) only three outcomes are possible, as outlined below. To be specific, we comment on the calculation of the right-hand derivative $\bar{\varphi}_{+}^{\prime}(0)$ using program (6). These comments equally apply to all special cases in which program (6) is replaced by simpler programs (8), (15) and (18).

Case 1. Let program (6) have a finite optimal solution. Then the unit $\left(X_{o}, Y_{o}\right)$ satisfies Assumption 1. (Otherwise, by statement 2 of Theorem 1, program (6) would be infeasible). Furthermore, $\gamma=0$ is not the right extreme point of the domain $\Gamma$. (Otherwise, by statement 1(ii), program (6) would have an unbounded optimal value.) This means it is possible to change the inputs and outputs of the unit $\left(X_{o}, Y_{o}\right)$ included in the set $A$ in the direction of vector $\Delta^{A}$. By statement 1(i), the directional derivative $\bar{\varphi}_{+}^{\prime}(0)$ exists and is equal to the optimal value of program (6).

Case 2. Let program (6) have an unbounded optimal value. As in Case 1, the unit $\left(X_{o}, Y_{o}\right)$ satisfies Assumption 1. However, unlike in Case 1, it is not possible within technology $\mathcal{T}$ to change the inputs and outputs of the unit $\left(X_{o}, Y_{o}\right)$ included in the set $A$ in the direction of vector $\Delta^{A}$. (Formally, $\gamma=0$ is the right extreme point of the domain $\Gamma$.) Because of this, the right-hand derivative $\bar{\varphi}_{+}^{\prime}(0)$ at the unit $\left(X_{o}, Y_{o}\right)$ is undefined.

Case 3. Let program (6) be infeasible. By statement 2 of Theorem 1, this means that the unit $\left(X_{o}, Y_{o}\right)$ does not satisfy Assumption 1 and the notion of directional derivative is therefore undefined. (For example, this case arises if the unit $\left(X_{o}, Y_{o}\right)$ is an interior point of technology $\mathcal{T}$.) 
Podinovski et al.: Marginal Values for Production Frontiers

Article submitted to Operations Research; manuscript no. (Please, provide the mansucript number!)

\section{Numerical Examples}

We illustrate how our methods can be applied by deriving a subset of the measures developed above in two commonly used polyhedral DEA technologies. Because these are not the standard VRS and CRS technologies, the known programming approaches of Podinovski and Førsund (2010) and Atici and Podinovski (2012) are not suitable for our task. Instead of the latter, we use the more general Theorem 1 and its variants applicable to any polyhedral technology.

\subsection{Scale Elasticity in a VRS Technology with Weight Restrictions}

Weight restrictions are often used in CRS and VRS DEA models in order to improve their discrimination (see, e.g., Thanassoulis et al. 2008). Such restrictions are incorporated in multiplier models as additional constraints on the vectors of input and output weights (shadow prices) $v \in \mathbb{R}_{+}^{m}$ and $u \in \mathbb{R}_{+}^{s}$.

Consider $K \geq 1$ weight restrictions in the form

$$
P_{t}^{\top} v-Q_{t}^{\top} u \geq 0, \quad t=1, \ldots, K
$$

where $P_{t} \in \mathbb{R}^{m}$ and $Q_{t} \in \mathbb{R}^{s}, \forall t$. Components of vectors $P_{t}$ and $Q_{t}$ can be positive, negative or zero. By duality, weight restrictions (26) generate the corresponding dual terms in the envelopment model called production trade-offs by Podinovski (2004b). The latter are interpretable as simultaneous changes to the inputs and outputs that apply to all units in the technology. As a result, the units in a VRS or CRS model with weight restrictions (26) are benchmarked against the frontier of the technology expanded by the dual production trade-offs.

Because the cases of VRS and CRS are similar, below we consider only the former case. Following Podinovski (2004b), the expanded technology $\mathcal{T}_{\text {VRS-TO }}$ is stated as the set of all nonnegative units $(X, Y)$ for which there exist intensity vectors $\lambda \in \mathbb{R}_{+}^{n}$ and $\pi \in \mathbb{R}_{+}^{K}$, and slack vectors $S_{X} \in \mathbb{R}_{+}^{m}$ and $S_{Y} \in \mathbb{R}_{+}^{s}$, such that

$$
\begin{aligned}
& \bar{X} \lambda+\bar{P} \pi+S_{X}=X, \\
& \bar{Y} \lambda+\bar{Q} \pi-S_{Y}=Y, \\
& 1^{\top} \lambda=1 .
\end{aligned}
$$

In the above formulation, $\bar{X}$ and $\bar{Y}$ are $m \times n$ and $s \times n$ matrices whose columns are, respectively, the input and output vectors $X_{j}$ and $Y_{j}$ of the observed units $j=1, \ldots, n$. Similarly, $\bar{P}$ and $\bar{Q}$ are $m \times K$ and $s \times K$ matrices whose columns are, respectively, the input and output trade-off vectors $P_{t}$ and $Q_{t}$ generated by weight restrictions (26).

Technology $\mathcal{T}_{\text {VRS-TO }}$ stated by conditions $(27)$ is a special case of polyhedral technology stated by conditions (1) and (2). This allows us to use the general methods developed above for the 
Table 1 One-sided scale elasticities in the VRS technology with weight restrictions $\mathcal{T}_{\text {VRS-TO. Where the scale }}$ elasticity is undefined, the table shows the solver diagnostic for the corresponding program (18) or (19).

\begin{tabular}{llllll}
\hline Unit & Input & Output 1 & Output 2 & $\varepsilon_{I, O}^{+}$ & $\varepsilon_{I, O}^{-}$ \\
\hline$F$ & 1 & 1 & 1 & 1.5 & $+\infty$ \\
$G$ & 2 & 3 & 2 & 0 & 1.25 \\
$H$ & 1.5 & 2 & 1.5 & 1.286 & 1.364 \\
$L$ & 1 & 0 & 2 & 1.5 & $+\infty$ \\
$M$ & 1 & 1.5 & 0 & 1.667 & $+\infty$ \\
$N$ & 2 & 3 & 1 & infeasible & infeasible \\
\hline
\end{tabular}

calculation of various marginal values on its frontier. Below we use a numerical example to illustrate the calculation of one-sided scale elasticities in this technology and the resulting characterization of RTS.

Consider the VRS technology with a single input and two outputs generated by two observed units $F$ and $G$ shown in Table 1. (The unobserved units $H, L, M$ and $N$ are also used in the discussion below.) Suppose we have specified two restrictions on the weights $u_{1}$ and $u_{2}$ of outputs 1 and 2 as follows:

$$
u_{1}-u_{2} \geq 0, \quad-0.5 u_{1}+u_{2} \geq 0
$$

In this case conditions (27) are stated as follows:

$$
\begin{aligned}
& 1 \lambda_{1}+2 \lambda_{2}+S_{X}^{1}=X_{1}, \\
& 1 \lambda_{1}+3 \lambda_{2}-1 \pi_{1}+0.5 \pi_{2}-S_{Y}^{1}=Y_{1}, \\
& 1 \lambda_{1}+2 \lambda_{2}+1 \pi_{1}-1 \pi_{2}-S_{Y}^{2}=Y_{2}, \\
& 1 \lambda_{1}+1 \lambda_{2}=1 .
\end{aligned}
$$

In the above formulation, the intensity variables $\lambda_{1}$ and $\lambda_{2}$ correspond to units $F$ and $G$, respectively. The terms $(-1,1)$ and $(0.5,-1)$ used in proportions $\pi_{1}$ and $\pi_{2}$ are the two output trade-offs generated by weight restrictions (28).

The VRS technology (29) is shown as the shaded polyhedral set in Figure 1. For example, unit $L$ satisfies (29) with $\lambda_{1}=\pi_{1}=1$, while all the other variables in (29) are equal to zero. Similarly, unit $M$ is obtained with $\lambda_{1}=\pi_{2}=1$, by taking the other variables equal to zero.

The one-sided scale elasticities $\varepsilon_{I, O}^{+}$and $\varepsilon_{I, O}^{-}$in technology (29) can be found using programs (18) and (19), by following the procedure discussed in $\S 5$. For example, program (18) for the calculation 


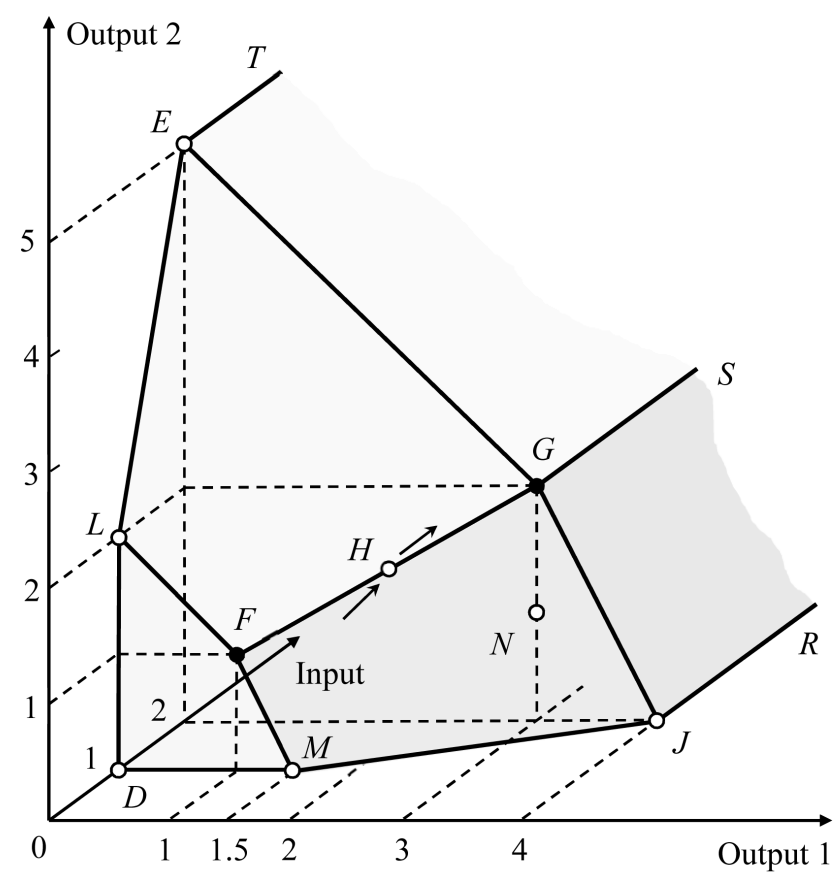

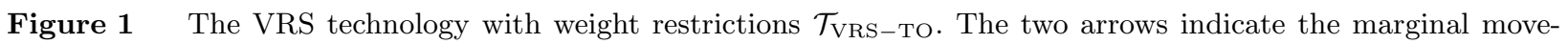
ments on the frontier corresponding to the calculation of the left and right-hand scale elasticity at point $H$.

of the right-hand scale elasticity at unit $G$ takes on the following form:

$$
\begin{aligned}
\varepsilon_{I, O}^{+}= & \min \quad 2 \nu_{1} \\
\text { subject to } & 3 \mu_{1}+2 \mu_{2}=1, \\
& 2 \nu_{1}-3 \mu_{1}-2 \mu_{2}+\omega=0, \\
& \nu_{1}-\mu_{1}-\mu_{2}+\omega \geq 0, \\
& 2 \nu_{1}-3 \mu_{1}-2 \mu_{2}+\omega \geq 0, \\
& \mu_{1}-\mu_{2} \geq 0 \\
& -0.5 \mu_{1}+\mu_{2} \geq 0, \\
& \nu_{1}, \mu_{1}, \mu_{2} \geq 0, \omega \operatorname{sign} \text { free. }
\end{aligned}
$$

Table 1 shows the one-sided scale elasticities calculated at the observed units $F$ and $G$ and at further four unobserved units.

Several observations are worth highlighting. The right-hand scale elasticity $\varepsilon_{I, O}^{+}$at unit $F$ is equal to 1.5 and corresponds to a marginal movement away from it, keeping the resulting unit on the facet $E G F L .^{19}$ The left-hand scale elasticity $\varepsilon_{I, O}^{-}$at unit $F$ is undefined because any reduction of its input leads outside the technology. When we assess the undefined left-hand scale elasticity 
Table 2 One-sided partial scale elasticities in technology $\mathcal{T}_{\text {VRS-TO }}$ and related solver diagnostics.

\begin{tabular}{lll}
\hline Unit & $\varepsilon_{A, B}^{+}$ & $\varepsilon_{A, B}^{-}$ \\
\hline$F$ & 2.5 & $+\infty$ \\
$G$ & 0 & 2 \\
$H$ & 1.875 & 2.25 \\
$L$ & infeasible & infeasible \\
$M$ & 1.667 & $+\infty$ \\
$N$ & infeasible & infeasible \\
\hline
\end{tabular}

at unit $F$ by solving program (19), which in our case is program (30) whose objective function is maximized, we obtain an unbounded optimal value. Similar analysis applies to units $L$ and $M$.

In the case of units $G$ and $H$, both one-sided scale elasticities are finite. The last unit $N$ is output radial inefficient and does not satisfy Assumption 2. Consequently, the one-sided scale elasticities are undefined at $N$. Both programs (18) and (19) stated for this unit are infeasible.

According to Definition 3, units $F, H, L$ and $M$ exhibit IRS. Unit $G$ exhibits CRS. Finally, unit $N$ does not satisfy Assumption 2, and the definition of RTS does not apply to it.

\subsection{Partial Scale Elasticity}

Consider the same technology $\mathcal{T}_{\mathrm{VRS}-\mathrm{TO}}$ as in Figure 1 . Assume that output 1 is discretionary and can be changed in the short run scenario, while output 2 is either exogenously fixed or can be changed only in the long run. In this setting we may be interested in the partial scale elasticity representing the response of output 1 to marginal changes of the input, while keeping output 2 constant. This in turn leads to the characterization of partial RTS.

The above scenario is modeled by including the input in the set $A$, output 1 in the set $B$ and output 2 in the set $C$. The one-sided partial scale elasticities $\varepsilon_{A, B}^{+}$and $\varepsilon_{A, B}^{-}$are calculated by appropriately specified programs (15) and (16), respectively. For example, the right-hand partial scale elasticity $\varepsilon_{A, B}^{+}$at unit $G$ is assessed by solving program (30) in which the first normalizing constraint is replaced by the equality $3 \mu_{1}=1$.

Table 2 shows the one-sided partial scale elasticities $\varepsilon_{A, B}^{+}$and $\varepsilon_{A, B}^{-}$at the selected units in the technology. Note that $\varepsilon_{A, B}^{+}$and $\varepsilon_{A, B}^{-}$are undefined at unit $L$, although $L$ is fully efficient. The reason of this is that output 1 of unit $L$ is equal to zero, and Assumption 2 is not satisfied. By Theorem 1 and Proposition 5, the corresponding linear programs (15) and (16) are infeasible, which is observed by computations and shown in Table 2 .

Based on the one-sided partial scale elasticities, we conclude that, in terms of the partial RTS characterization, units $F, H$ and $M$ exhibit IRS, unit $G$ exhibits CRS, and this notion is undefined 
at units $L$ and $N$. The interpretation of this characterization is similar to the conventional notion of RTS. For example, because unit $F$ exhibits IRS, a marginal increase of its input would lead to a more than proportional increase of output 1, provided output 2 is kept unchanged. More precisely, observing that $\varepsilon_{A, B}^{+}=2.5$, a $1 \%$ increase of the input would result in a $2.5 \%$ increase of output 1 .

\subsection{Marginal Rates in a Weakly Disposable VRS Technology}

In this example we consider the polyhedral technology $\mathcal{T}_{K}$ introduced by Kuosmanen (2005). Similar to the Shephard technology (Shephard 1974), technology $\mathcal{T}_{K}$ exhibits weak disposability of undesirable outputs with respect to good outputs. ${ }^{20}$ As shown by Kuosmanen and Podinovski (2009), the Shephard technology is generally not convex, and technology $\mathcal{T}_{K}$ is its convex hull.

In the described setting, the vector of outputs is split into the subvectors $V$ and $W$ of good and bad (undesirable) outputs, respectively: $Y=(V, W)$. Following Kuosmanen (2005), technology $\mathcal{T}_{K}$ can be stated as the set of all nonnegative units $(X, V, W)$ for which there exist intensity vectors $\lambda, \mu \in \mathbb{R}_{+}^{n}$ and nonnegative slack vectors of input $S_{X}$ and good output $S_{V}$ of appropriate dimensions such that the following conditions are true:

$$
\begin{aligned}
& \bar{X}(\lambda+\mu)+S_{X}=X, \\
& \bar{V} \lambda-S_{V}=V, \\
& \bar{W} \lambda=W, \\
& 1^{\top}(\lambda+\mu)=1 .
\end{aligned}
$$

In the above statement, $\bar{X}, \bar{V}$ and $\bar{W}$ are the matrices of inputs, and good and bad outputs of appropriate dimensions, respectively.

Table 3 Marginal rates of transformation of good output 1 with respect to undesirable output 2 and related solver diagnostics.

\begin{tabular}{llllll}
\hline Unit & Input & Output 1 & Output 2 & $\frac{\partial^{+} V}{\partial W}$ & $\frac{\partial^{-} V}{\partial W}$ \\
\hline$F$ & 1 & 1 & 1 & $-\infty$ & 1 \\
$G$ & 2 & 1.5 & 2 & $-\infty$ & 0.5 \\
$H$ & 2 & 1 & 1 & 0.5 & 1 \\
$L$ & 1 & 0 & 0 & 1 & $+\infty$ \\
$M$ & 2 & 1 & 2 & infeasible & infeasible \\
\hline
\end{tabular}




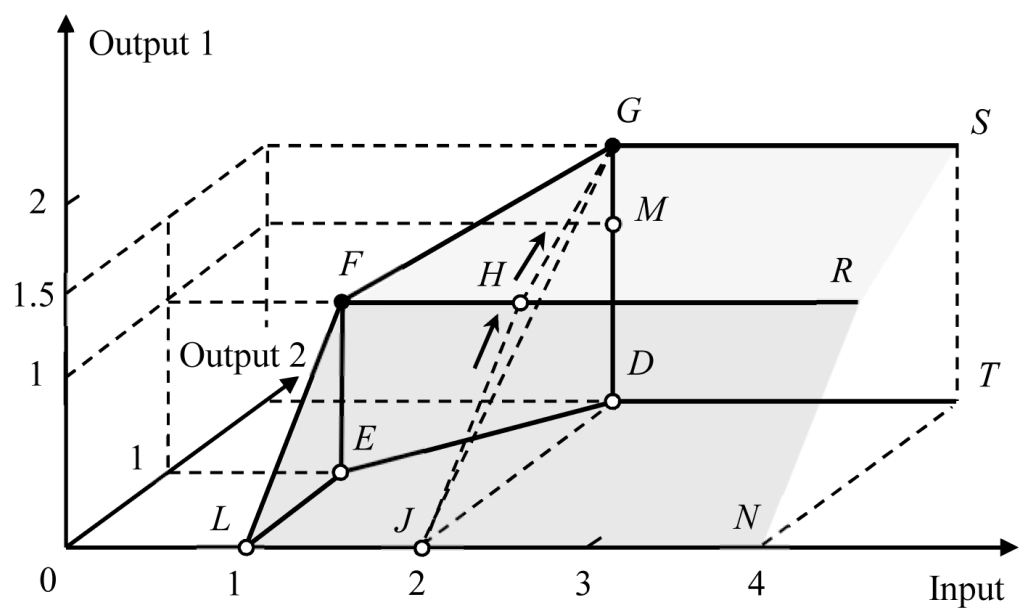

Figure 2 Technology $\mathcal{T}_{K}$ with undesirable output 2 assumed weakly disposable with respect to good output 1. The two arrows indicate the marginal movements on the frontier corresponding to the calculation of the left and right-hand marginal rates of transformation of output 1 with respect to output 2 at point $H$.

Consider technology $\mathcal{T}_{K}$ generated by two observed units $F$ and $G$ in Table 3 . In this example we assume that output 1 is good and output 2 is undesirable. Then conditions (31) describing technology $\mathcal{T}_{K}$ are stated as follows:

$$
\begin{aligned}
& 1\left(\lambda_{1}+\mu_{1}\right)+2\left(\lambda_{2}+\mu_{2}\right)+S_{X}=X, \\
& 1 \lambda_{1}+1.5 \lambda_{2}-S_{V}=V, \\
& 1 \lambda_{1}+2 \lambda_{2}=W, \\
& 1 \lambda_{1}+1 \lambda_{2}+1 \mu_{1}+1 \mu_{2}=1 .
\end{aligned}
$$

Technology $\mathcal{T}_{K}$ is shown as the shaded polyhedral set in Figure 2. (Observe that, by conditions (32), technology $\mathcal{T}_{K}$ is the convex hull of the four units: $F, G, J$ and $L$ shown in Figure 2, which is further freely disposed with respect to the input and good output 1.) ${ }^{21}$

Suppose we wish to assess the one-sided marginal rates of transformation (MRT) of the good output 1 with respect to the undesirable output 2, at the units $F, G$ and the unobserved units $H, L$ and $M$ defined in Table 3 . As shown in $\S 4.2$, the required MRT can be computed by solving appropriately specified general programs (6) and (7). For example, for unit $F$ program (6) takes on the following form (as noted in $\S 2.5$, the multiplier $\mu_{2}$ corresponding to output 2 is sign free): 


$$
\begin{aligned}
\left.\frac{\partial^{+} V}{\partial W}\right|_{\left(X_{o}, Y_{o}\right)=F}= & \min \quad-\mu_{2}+\sigma \\
\text { subject to } & \mu_{1}-\zeta=1, \\
& \nu_{1}-\mu_{1}-\mu_{2}+\omega+\sigma+\zeta=0, \\
& \nu_{1}-\mu_{1}-\mu_{2}+\omega \geq 0, \\
& \nu_{1}+\omega \geq 0, \\
& 2 \nu_{1}-1.5 \mu_{1}-2 \mu_{2}+\omega \geq 0, \\
& 2 \nu_{1}+\omega \geq 0, \\
& \nu_{1}, \mu_{1}, \sigma, \zeta \geq 0, \mu_{2}, \omega \text { sign free. }
\end{aligned}
$$

Note that, as shown in $\S 4.2$, because the input and both outputs of unit $F$ are strictly positive, we can replace program (33) by the simplified program (24), i.e., remove the variables $\sigma$ and $\zeta$ from (33). Restating program (33) for the other units is straightforward: we need to replace only the second constraint. For example, for unit $G$ this is replaced by the equality

$$
2 \nu_{1}-1.5 \mu_{1}-2 \mu_{2}+\omega+2 \sigma+1.5 \zeta=0 .
$$

Table 3 shows the results of computations. As in the previous example, the unbounded optimal value indicates that the specified MRT is undefined because the required perturbation of the bad output cannot be performed in the technology. For example, as seen from Figure 2, at units $F$ and $G$ an increase of the bad output 2 is impossible and leads outside the model of technology. Similarly, at unit $L$ a reduction of the bad output 2 is impossible.

At unit $H$ both one-sided marginal rates are defined and coincide with the slopes of lines $J H$ and $H G$, respectively. Finally, unit $M$ does not satisfy Assumption 1 (it does not produce the maximum amount of output 1 for the given levels of the input and output 2). Consequently, at this unit, the specified notion of MRT is undefined and the corresponding programs (6) and (7) are infeasible.

\section{Conclusion}

In this paper we present a unifying linear programming approach to the calculation of various directional marginal values for DEA production frontiers. Our development generalizes the earlier results of Podinovski and Førsund (2010), extends them to all polyhedral technologies and is unifying in several respects.

First, our approach applies to a very large class of all polyhedral technologies, including the standard VRS and CRS technologies, their variants with weight restrictions and production tradeoffs, weakly disposable technologies and many other. Second, it is suitable for the calculation of 
various marginal rates and elasticity measures evaluated in arbitrary directions, as special cases of the single general formulation. Third, it applies to the entire production frontier, including all extreme points of the polyhedral technology that typically correspond to observed units. Fourth, it provides a general method for the RTS characterization of units in any polyhedral technology. This effectively removes the necessity to develop special methods of RTS characterization for each DEA technology.

With few exceptions, the existing methods for the calculation of scale elasticity and marginal rates are applicable only to the conventional VRS and CRS technologies. The suggested approach allows us to calculate all these marginal characteristics in any polyhedral technology, by solving essentially the same linear programs. The latter obviously need to be appropriately specified for the technology and marginal value under the consideration, which is an uncomplicated task.

An important special case of our development is the RTS characterization of efficient frontiers of any polyhedral technology. In our approach the RTS types (increasing, decreasing and constant) are defined based on the underlying notion of one-sided scale elasticity. This contrasts with some other developments in which the RTS characterization is either postulated or computed without a reference to the scale elasticity, which remains undefined in such approaches.

The practical use of the presented approach is straightforward. The linear programs developed in our paper can be solved in a single batch on the entire set of all observed units. There is no need to restrict the computations only to efficient units for which the selected marginal perturbation is feasible. All inefficient units and infeasibility problems are automatically, according to our main theorem, diagnosed by the standard output produced by linear optimizers. We illustrate this approach by several numerical examples. 
Podinovski et al.: Marginal Values for Production Frontiers

Article submitted to Operations Research; manuscript no. (Please, provide the mansucript number!)

\section{Appendix A. Proofs}

Proof of Proposition 1. By definition (Rockafellar 1970), the set $W$ of all solutions $\left(X, Y, \hat{\lambda}, S_{X}, S_{Y}\right)$ to the set of linear equalities and inequalities (1) and (2) is a polyhedral set. Then technology $\mathcal{T}$ is the projection of $W$ on the input and output dimensions $X$ and $Y$, i.e., $\mathcal{T}$ is the set of all pairs $(X, Y)$ for which there exist $\hat{\lambda}, S_{X}$ and $S_{Y}$ such that $\left(X, Y, \hat{\lambda}, S_{X}, S_{Y}\right) \in W$. By the projection lemma (see, e.g., Jones et al. 2008, Lemma 3.1), $\mathcal{T}$ is a polyhedral set.

Proof of Proposition 2. The optimal value $\bar{\varphi}(\gamma)$ of program (5) is a function of parameter $\gamma$ attached to the perturbation vector $\Delta^{A}$. Then the proof follows from the maximization analogues of Theorems IV.48 and IV.50 in Roos et al. (2005).

Proof of Theorem 1. Consider the dual to program (5), where $\gamma=0$ :

$$
\bar{\varphi}(0)=\min \quad X_{o}^{\top} \nu-Y_{o}^{\top} \mu+U_{o}^{\top} \omega+\left(X_{o}^{A}\right)^{\top} \rho+\left(Y_{o}^{A}\right)^{\top} \sigma+\left(X_{o}^{B}\right)^{\top} \xi+\left(Y_{o}^{B}\right)^{\top} \zeta
$$

subject to conditions $(6.2),(6.4),(6.5)$.

Let Assumption 1 be true. Then $\bar{\varphi}(0)=0$. Denote $\mathcal{D}^{*}$ the set of optimal solutions to program (34). Note that $\mathcal{D}^{*} \neq \varnothing$. The proof of statements 1(i)-(iv) is based on the theorem of marginal values in linear programming, stated as Theorem IV.62 in Roos et al. (2005). (We use its maximization analogue.) To prove 1(i), suppose that $\gamma$ is not the right extreme point of $\Gamma$. Then, by the theorem of marginal values, $\bar{\varphi}_{+}^{\prime}(0)$ exists, is finite and

$$
\begin{aligned}
& \bar{\varphi}_{+}^{\prime}(0)=\min \left(\Delta_{X}^{A}\right)^{\top} \nu^{A}-\left(\Delta_{Y}^{A}\right)^{\top} \mu^{A}+\left(\Delta_{X}^{A}\right)^{\top} \rho+\left(\Delta_{Y}^{A}\right)^{\top} \sigma \\
& \text { subject to }(\mu, \nu, \rho, \sigma, \xi, \zeta, \omega) \in \mathcal{D}^{*} .
\end{aligned}
$$

The constraints of program (35) are the same as in (34), with the additional condition that the objective function of (34) is equal to zero, stated as (6.3). Therefore, program (35) is program (6).

To prove 1(ii), let $\gamma$ be the right extreme point of $\Gamma$. By Theorem IV.62 in Roos et al. (2005), program (35), and therefore (6), is unbounded. The proof of statements 1(iii) and 1(iv) is similar. By the theorem of marginal values, $\bar{\varphi}_{-}^{\prime}(0)$ is obtained by changing the minimization in program (35) to maximization.

Finally, suppose that Assumption 1 is not true. Two possibilities arise. If the maximum in (4) is unbounded (see the discussion in Example 1 in $\S 2.2$ ), then the optimal value of program (5) at $\gamma=0$ is also unbounded, and the dual optimal set $\mathcal{D}^{*}=\varnothing$. Therefore programs (6) and (7) are infeasible. Alternatively, let the maximum in (4) be finite. Then $\bar{\varphi}(0)>0$ because $\varphi=0$ is feasible with $\gamma=0$ in program (5). Then for any dual optimal solution in $\mathcal{D}^{*}$, the objective function of program (34) is equal to $\bar{\varphi}(0)>0$. Therefore, equality (6.3) cannot be satisfied on the set $\mathcal{D}^{*}$, and programs (6) and (7) are infeasible. 
Lemma 1. In the case 1(i) of Theorem 1, any optimal solution to program (6), and in the case case 1(iii), any optimal solution to program (7), satisfies the conditions:

$$
\begin{aligned}
& \left(X_{o}^{A}\right)_{i} \rho_{i}=0, \quad \forall i \in A, \\
& \left(Y_{o}^{A}\right)_{r} \sigma_{r}=0, \quad \forall r \in A, \\
& \left(X_{o}^{B}\right)_{i} \xi_{i}=0, \quad \forall i \in B, \\
& \left(Y_{o}^{B}\right)_{r} \zeta_{r}=0, \quad \forall r \in B .
\end{aligned}
$$

Proof of Lemma 1. Any optimal solution to programs (6) and (7) is in the set $\mathcal{D}^{*}$ of optimal solutions to program (34), which is the dual to (5) with $\gamma=0$. It suffices to prove that any $\langle\mu, \nu, \rho, \sigma, \xi, \zeta, \omega\rangle \in \mathcal{D}^{*}$ satisfies (36). Indeed, the dual inequality to variable $\left(D_{X}^{A}\right)_{i}$ is $\rho_{i} \geq 0$. By complementary slackness, at optimality, $\left(D_{X}^{A}\right)_{i} \rho_{i}=0$. From (5.9) with $\gamma=0,\left(D_{X}^{A}\right)_{i}=\left(X_{o}^{A}\right)_{i}$, and the first equality in (36) follows. The proof of the remaining equalities in (36) is similar.

Proof of Proposition 4. Consider statement 1(i) of Theorem 1. Because $X_{o}^{A}, Y_{o}^{A}, X_{o}^{B}, Y_{o}^{B}>0$ and by (36), at any optimal solution to $(6), \rho, \sigma, \xi$ and $\zeta$ are zero vectors. Therefore, the optimal values of programs (6) and (8) are equal, and the statement 1(i) follows.

For the further proof, let $\tilde{\mathcal{T}}$ be the technology defined by conditions (1) only, without the nonnegativity conditions $(2)$. Obviously, $\left(X_{o}, Y_{o}\right) \in \tilde{\mathcal{T}}$. The corresponding directional response function $\tilde{\varphi}(\gamma)$ is defined by program (5) from which the constraints (5.9)-(5.12) and vectors $D_{X}^{A}, D_{Y}^{A}, D_{X}^{B}$ and $D_{Y}^{B}$ are omitted. Denote the resulting program $\tilde{\mathbb{P}}$. Let $\tilde{\Gamma}$ be the domain of the function $\tilde{\varphi}(\gamma)$. Note that programs (6) and (7) in Theorem 1 stated for the one-sided derivatives of the function $\tilde{\varphi}(\gamma)$ are, respectively, programs (8) and (9).

Consider statement 1(ii) of Theorem 1. We need to prove that $\gamma=0$ is the right extreme point of $\tilde{\Gamma}$ which would imply that program (8) has an unbounded optimal value. Assume that, on the contrary, there exists a feasible solution $\Psi^{1}=\left\langle\hat{\lambda}^{1}, \gamma^{1}, \varphi^{1}, S_{X}^{1}, S_{Y}^{1}\right\rangle$ to program $\tilde{\mathbb{P}}$, where $\gamma^{1}>0$. Because $\left(X_{o}, Y_{o}\right)$ satisfies Assumption 1, there exists an optimal solution to program (5) in the form $\Psi^{2}=\left\langle\hat{\lambda}^{2}, \gamma^{2}, \varphi^{2}, S_{X}^{2}, S_{Y}^{2}\right\rangle$, where $\gamma^{2}=\varphi^{2}=0$. Note that $\Psi^{2}$ is feasible in program $\tilde{\mathbb{P}}$. Then for any $\delta \in[0,1], \Psi(\delta)=\delta \Psi^{1}+(1-\delta) \Psi^{2}$ is feasible in $\tilde{\mathbb{P}}$. Note that in $\Psi(\delta), \gamma(\delta)=\delta \gamma^{1}+(1-\delta) \gamma^{2}=\delta \gamma^{1}$ and, similarly, $\varphi(\delta)=\delta \varphi^{1}$. Because $X_{o}^{A}, Y_{o}^{A}, X_{o}^{B}, Y_{o}^{B}>0$, for all sufficiently small $\delta>0, \Psi(\delta)$ satisfies conditions (5.9)-(5.12), together with some vectors $D_{X}^{A}, D_{Y}^{A}, D_{X}^{B}, D_{Y}^{B} \geq 0$. This contradicts the assumption that $\gamma=0$ is the right extreme point of $\Gamma$. Therefore, $\gamma=0$ is the right extreme point of $\tilde{\Gamma}$, and the statement 1(ii) follows.

Consider statement 2 of Theorem 1, and let program (6) be infeasible. Then program (8) is also infeasible. Indeed, if there exists a feasible solution $\mu^{*}, \nu^{*}$ and $\omega^{*}$ to $(8)$, then the same solution is feasible in (6), together with the zero vectors $\rho, \sigma, \xi$ and $\zeta$.

The proof that (7) can be substituted by (9) is similar. 
Proof of Proposition 5. In statement 1(i) of Theorem 1, let $\langle\mu, \nu, \omega, \rho, \sigma, \xi, \zeta\rangle$ be any optimal solution to (6). By (36) and (12), $\langle\mu, \nu, \omega\rangle$ is feasible and optimal in (15).

The statement 1 (ii) repeats the proof of Proposition 4 for the same case, with the following modification. By conditions (12), the feasible solution $\Psi(\delta)$ of program $\tilde{\mathbb{P}}$ also satisfies conditions (5.9)-(5.12), together with some vectors $D_{X}^{A}, D_{Y}^{A}, D_{X}^{B}, D_{Y}^{B} \geq 0$, for all sufficiently small $\delta>0$. More precisely, we can take any $\delta$ such that $1+\delta \gamma^{1} \geq 0$ and $1+\delta \varphi^{1} \geq 0$. For all such $\delta$ the expressions on the right-hand side of conditions (5.9)-(5.12) are nonnegative. For example, in the case of (5.9), taking into account that $\Delta_{X}^{A}=X_{o}^{A}$, we have $X_{o}^{A}+\gamma^{1} \Delta_{X}^{A}=\left(1+\delta \gamma^{1}\right) X_{o}^{A} \geq 0$.

The proof in the case of statement 2 of Theorem 1 is the same as in Proposition 4. The proof that $(7)$ can be substituted by (16) is similar. 


\section{Endnotes}

1. Atici and Podinovski (2015) demonstrate the use of the VRS and CRS technologies extended by production trade-offs in the context of efficiency assessment of agricultural farms. The notions of elasticity measures and RTS are left outside the scope of this paper.

2. The specification of conditions (1.3) as equality is not restrictive. An inequality can be converted to equality by introducing a nonnegative slack variable. For example, the condition $1^{\top} \lambda \leq 1$ used in the definition of the non-increasing returns-to-scale technology is restated as $1^{\top} \lambda+\lambda_{0}=1$, where $\lambda_{0} \geq 0$. In this case $\hat{\lambda}=\left(\lambda, \lambda_{0}\right)$. Alternatively, if (1.3) allows inequalities, the corresponding components of the dual vector $\omega$ in programs (6) and (7) should be of the appropriate sign. Similarly, the condition $\hat{\lambda} \geq 0$ in (1.4) is not restrictive.

3. If some inputs or outputs are not required to be nonnegative, the corresponding inequalities in conditions (2) are omitted.

4. Under the assumption of free disposability, $\bar{\varphi}(0)$ is a complete function representation of $\mathcal{T}$ (Chambers et al. 1998).

5. Assumption 1 accords with a standard directional-based definition of efficiency (Chambers et al. 1998). We use the term weak efficiency because equality (4) may still allow changes of the unit $\left(X_{o}, Y_{o}\right)$ in the direction of individual components of vector $\Delta^{B}$. If the unit $\left(X_{o}, Y_{o}\right)$ satisfies Assumption 1, then it is weakly efficient in the conventional sense, but the converse is not true.

6. The difference $\bar{\varphi}(\gamma)-\bar{\varphi}(0)$ is interpretable as the difference between a directional distance function evaluated at $\left(X_{o}^{A}+\gamma \Delta_{X}^{A}, X_{o}^{B}, X_{o}^{C}, Y_{o}^{A}+\gamma \Delta_{Y}^{A}, Y_{o}^{B}, Y_{o}^{C}\right)$ and $\left(X_{o}, Y_{o}\right)$. Hence, for example, the right-hand derivative $\varphi_{+}^{\prime}(0)$ corresponds to the directional derivative of the type defined by Rockafellar (1970) for the directional distance function evaluated at $\left(X_{o}, Y_{o}\right)$ in the direction $\left(\Delta_{X}^{A}, 0,0, \Delta_{Y}^{A}, 0,0\right)$. Chambers and Färe (2008) use these directional derivatives in their development of a "calculus" for DEA technologies.

7. From (5.2)-(5.4), the dual constraint corresponding to the primal vector $S_{X}$ is $\nu \geq 0$. Similarly, from (5.5)-(5.7), the dual constraint to the primal vector $S_{Y}$ is $-\tilde{\mu} \geq 0$. Then $\mu=-\tilde{\mu} \geq 0$.

8. Let us show that any polyhedral technology $\mathcal{T}$ can be stated in the form (1) from which the slack vectors $S_{X}$ and $S_{Y}$ are omitted. By definition of a polyhedral set (Rockafellar 1970), any such technology $\mathcal{T}$ is the set of all pairs $Z=(X, Y) \in \mathbb{R}^{m+s}$ that satisfy some vector inequality $F Z \leq d$, where matrix $F$ is of dimension $p \times(m+s)$ and $d \in \mathbb{R}^{p}$. This can be restated by the three equalities as follows: $\chi=X, \tau=Y$, and $F(\chi, \tau)+\delta=d$, where $\chi, \tau$ and $\delta$ are variable vectors, and $\delta \geq 0$. This satisfies the description (1.1)-(1.3) from which the slack vectors $S_{X}$ and $S_{Y}$ are omitted, and where $\hat{\lambda}=(\chi, \tau, \delta)$. Finally, condition (1.4) is satisfied by the standard substitution $\chi=\chi_{1}-\chi_{2}$ and $\tau=\tau_{1}-\tau_{2}$, where $\chi_{1}, \chi_{2}, \tau_{1}$ and $\tau_{2}$ are nonnegative vectors. 
Podinovski et al.: Marginal Values for Production Frontiers

9. If $\mathcal{T}$ satisfies free disposability in at least one input or output, $\Delta^{B}$ can be chosen to ensure that $\bar{\varphi}(0)$ and $\bar{\varphi}(\gamma)$ represent directional distance functions, evaluated at different points, that fully characterize $\mathcal{T}$. Regardless of the disposability properties imposed upon $\mathcal{T}$, it is well-known that the directional distance function corresponding to $\bar{\varphi}(0)$ is concave in $\left(X_{o}, Y_{o}\right)$ which implies that $\bar{\varphi}(\gamma)$ is concave as a function of $\gamma$. Under Assumption 1, $\bar{\varphi}_{+}^{\prime}(0)$ is positively linearly homogeneous, concave, closed, and proper as a function of $\left(\Delta_{X}^{A}, 0,0, \Delta_{Y}^{A}, 0,0\right)$ (Rockafellar 1970, Theorem 23.4).

10. Note that, similarly to $\bar{\varphi}(0), \bar{\beta}(1)$ can also be recognized as a distance or, more properly, a gauge function. Specifically, $\bar{\beta}(1)$ is the reciprocal of the (partial) gauge function

$$
\bar{\psi}(1)=\inf \left\{\psi \mid\left(X_{o}^{A}, \frac{X_{o}^{B}}{\psi}, X_{o}^{C}, Y_{o}^{A}, \frac{Y_{o}^{B}}{\psi}, Y_{o}^{C}\right) \in \mathcal{T}, \psi>0\right\},
$$

for $\mathcal{T}$ evaluated at $\left(X_{o}, Y_{o}\right)$ (Rockafellar 1970, p. 28). Under weak disposability, both $\bar{\psi}(1)$ and therefore $\bar{\beta}(1)$ are complete function representations of $\mathcal{T}$. Furthermore, $\bar{\beta}(\alpha)$ is interpretable as the reciprocal of the gauge function evaluated at $\left(\alpha X_{o}^{A}, X_{o}^{B}, X_{o}^{C}, \alpha Y_{o}^{A}, Y_{o}^{B}, Y_{o}^{C}\right)$. Then $\bar{\beta}_{+}^{\prime}(1)=$ $\left(\bar{\psi}^{-1}\right)_{+}^{\prime}(1)$. Setting $\bar{\beta}(1)=1$ is equivalent to requiring that $\left(X_{o}, Y_{o}\right)$ satisfies a standard efficiency criterion.

11. In this case, Assumption 1 corresponds to the equivalent Assumption 2. Furthermore, the case of $\gamma=0$ being the right or left extreme point of $\Gamma$ corresponds to $\alpha=1$ being the right or, respectively, left extreme point of the domain $\Gamma^{*}$ of the function $\bar{\beta}(\alpha)$.

12. By Proposition 5, both one-sided elasticities exist and inequality (17) is true, if $\alpha=1$ is not an extreme point of the domain $\Gamma^{*}$ of the function $\bar{\beta}(\alpha)$.

13. Consider the output oriented envelopment model $\eta^{*}=\max \left\{\eta \mid\left(X_{o}, \eta Y_{o}\right) \in \mathcal{T}, \eta\right.$ sign free $\}$. Without loss of generality, let $\mathcal{T}$ be defined by conditions (1) only: conditions (2) are redundant for the radial output maximization, provided $\left(X_{o}, Y_{o}\right)$ satisfies $(2)$. The dual multiplier model minimizes $X_{o}^{\top} \nu+U_{o}^{\top} \omega$ subject to conditions (18.2), (18.4) and (18.5). Because $\left(X_{o}, Y_{o}\right)$ is assumed output radial efficient, at any optimal dual solution we have $X_{o}^{\top} \nu+U_{o}^{\top} \omega=1$. By (18.2), the latter equality is equivalent to (18.3). Therefore, the constraints (18.2)-(18.5) define the set $\Omega$.

14. The optimal dual vectors $\omega$ of the input-oriented multiplier model characterize the onesided inverse scale elasticities (Podinovski et al. 2009). If unit $\left(X_{o}, Y_{o}\right)$ is both input and output radial efficient, the latter can be inverted to obtain the corresponding one-sided scale elasticities. This leads to the formulae: $\varepsilon_{I, O}^{+}\left(X_{o}, Y_{o}\right)=1 /\left(1-\min _{\langle\mu, \nu, \omega\rangle \in \Delta}\left\{U_{o}^{\top} \omega\right\}\right)$ and $\varepsilon_{I, O}^{-}\left(X_{o}, Y_{o}\right)=$ $1 /\left(1-\max _{\langle\mu, \nu, \omega\rangle \in \Delta}\left\{U_{o}^{\top} \omega\right\}\right)$, where $\Delta$ is the set of optimal solutions to the input-oriented multiplier model. This generalizes formula (8) for the VRS technology given in Banker and Thrall (1992).

15. In economics, an efficient unit $\left(X_{o}, Y_{o}\right)$ exhibits CRS if a proportional change of inputs results in exactly the same proportional change of outputs. The case (c) of Definition 3 does not imply such proportionality, unless $\varepsilon_{I, O}^{+}\left(X_{o}, Y_{o}\right)=\varepsilon_{I, O}^{-}\left(X_{o}, Y_{o}\right)=1$. However, it is straightforward to verify that in case $(\mathrm{c})$ the unit $\left(X_{o}, Y_{o}\right)$ represents the most productive scale size (Banker 1984). 
16. Because in the standard VRS technology $X_{o} \geq 0$, the objective function of program (18) is nonnegative, and $\varepsilon_{I, O}^{+}\left(X_{o}, Y_{o}\right)$ is always finite. The objective function in (19) may be unbounded.

17. As noted in $\S 3.3$, the computational complexity of our approach is identical to the complexity of the standard method used in the VRS technology. The latter requires the calculation of the minimum and maximum values of the dual variable $\omega$ corresponding to the normalizing equality.

18. Similar to the last observation of Example 1, if we define $\left(\Delta_{X}^{B}\right)_{i^{*}}=1$, the maximum in (4) is unbounded and Assumption 1 is not satisfied.

19. This and further results are easy to verify using a standard formula for the scale elasticity evaluated on smooth frontiers - see, e.g., Førsund and Hjalmarsson (2004). For example, the plane EGFL satisfies the equation $\Phi\left(X_{1}, Y_{1}, Y_{2}\right)=3 X_{1}-Y_{1}-Y_{2}-1=0$. The scale elasticity at any point $\left(X_{o}, Y_{o}\right)$ on this plane is equal to $-\left\langle X_{o}, \nabla_{X} \Phi\left(X_{o}, Y_{o}\right)\right\rangle /\left\langle Y_{o}, \nabla_{Y} \Phi\left(X_{o}, Y_{o}\right)\right\rangle$, where $\nabla_{X} \Phi\left(X_{o}, Y_{o}\right)=(3)$ and $\nabla_{Y} \Phi\left(X_{o}, Y_{o}\right)=(-1,-1)^{\top}$. Applying the above formula to unit $F$, we obtain the value 1.5 , which is the same as the value $\varepsilon_{I, O}^{+}$at the unit $F$ in Table 1.

20. The assumption of weak disposability arises from the notion of null-jointness - see, e.g., the discussion in Färe and Grosskopf (2004).

21. It is straightforward to verify that, in this example, the Shephard technology is the same as technology $\mathcal{T}_{K}$ but, as noted, generally this is not so.

\section{Acknowledgments}

The authors acknowledge the support of the European Commission through the Seventh Framework Programme (FP7) project 265616, titled "Integrating econometric and mathematical programming models into an amendable policy and market analysis tool using FADN database". 
Podinovski et al.: Marginal Values for Production Frontiers

Article submitted to Operations Research; manuscript no. (Please, provide the mansucript number!)

\section{References}

Atici KB, Podinovski VV (2012) Mixed partial elasticities in constant returns-to-scale production technologies. Eur. J. Oper. Res. 220(1):262-269.

Atici KB, Podinovski VV (2015) Using data envelopment analysis for the assessment of technical efficiency of units with different specialisations: An application to agriculture. Omega 54:72-83.

Balk BM, Färe R, Karagiannis G (2015) On directional scale elasticities. J. Productivity Anal. 43(1):99-104.

Banker RD (1984) Estimating most productive scale size using data envelopment analysis. Eur. J. Oper. Res. 17(1):35-44.

Banker RD, Charnes A, Cooper WW (1984) Some models for estimating technical and scale efficiencies in data envelopment analysis. Management Sci. 30(9):1078-1092.

Banker RD, Cooper WW, Seiford LM, Zhu J (2011) Returns to scale in DEA. Cooper WW, Seiford LM, Zhu J, eds. Handbook on Data Envelopment Analysis. 2nd edition. (Springer Science+Business Media, New York) 41-70.

Banker RD, Maindiratta A (1986) Piecewise loglinear estimation of efficient production surfaces. Management Sci. 32(1):126-135.

Banker RD, Thrall RM (1992) Estimation of returns to scale using data envelopment analysis. Eur. J. Oper. Res. 62(1):74-84.

Chambers RG, Chung Y, Färe R (1998) Profit, directional distance functions, and Nerlovian efficiency. $J$. Optim. Theory Appl. 98(2):351-364.

Chambers RG, Färe R (2008) A “calculus" for data envelopment analysis. J. Productivity Anal. 30(3):169175.

Charnes A, Cooper WW, Golany B, Seiford L, Stutz J (1985) Foundations of data envelopment analysis for Pareto-Koopmans efficient empirical production functions. J. Econometrics 30(1-2):91-107.

Charnes A, Cooper WW, Rhodes E (1978) Measuring the efficiency of decision making units. Eur. J. Oper. Res. 2(6):429-444.

Chen C-M, Delmas MA (2012) Measuring eco-efficiency: A new frontier approach. Oper. Res. 60(5):10641079.

Cook WD, Zhu J (2011) Multiple variable proportionality in data envelopment analysis. Oper. Res. 59(4):1024-1032.

Cooper WW, Seiford LM, Tone K (2007) Data Envelopment Analysis. A Comprehensive Text with Models, Applications, References and DEA-Solver Software, 2nd ed. (Springer Science + Busines Media, New York).

Emrouznejad A, Anouze AL, Thanassoulis E (2010) A semi-oriented radial measure for measuring the efficiency of decision making units with negative data, using DEA. Eur. J. Oper. Res. 200(1):297-304. 
Färe R, Grosskopf S (1985) A nonparametric cost approach to scale efficiency. Scand. J. Economics 87(4):594604.

Färe R, Grosskopf S (2004) New Directions: Efficiency and Productivity (Kluwer Academic Publishers, Dordrecht, Netherlands).

Førsund FR, Hjalmarsson L (2004) Calculating scale elasticity in DEA models. J. Oper. Res. Soc. 55(10):1023-1038.

Fukuyama H (2000) Returns to scale and scale elasticity in data envelopment analysis. Eur. J. Oper. Res. 125(1):93-112.

Hadjicostas P, Soteriou AC (2006) One-sided elasticities and technical efficiency in multi-output production: A theoretical framework. Eur. J. Oper. Res. 168(2):425-449.

Jones CN, Kerrigan EC, Maciejowski JM (2008) On polyhedral projection and parametric programming. J. Optim. Theory Appl. 138(2):207-220.

Korhonen PJ, Soleimani-damaneh M, Wallenius J (2011) Ratio-based RTS determination in weight-restricted DEA models. Eur. J. Oper. Res. 215(2):431-438.

Krivonozhko VE, Førsund FR, Lychev AV (2014) Measurement of returns to scale using non-radial DEA models. Eur. J. Oper. Res. 232(3):664-670.

Krivonozhko VE, Utkin OB, Volodin AV, Sablin IA, Patrin M (2004) Constructions of economic functions and calculations of marginal rates in DEA using parametric optimization methods. J. Oper. Res. Soc. 55(10):1049-1058.

Kuosmanen T (2005) Weak disposability in nonparametric productivity analysis with undesirable outputs. Amer. J. Agricultural Econom. 87(4):1077-1082.

Kuosmanen T, Podinovski VV (2009) Weak disposability in nonparametric production analysis: Reply to Färe and Grosskopf. Amer. J. Agricultural Econom. 91(2):539-545.

Mills HD (1952) Marginal values of matrix games and linear programs. Kuhn HW, Tucker AW, eds. Linear Equalities and Related Systems (Princeton University Press, Princeton, NJ) 183-193.

Olesen OB, Petersen NC (1996) Indicators of ill-conditioned data sets and model misspecification in data envelopment analysis: An extended facet approach. Management Sci. 42(2):205-219.

Podinovski VV (2004a) Bridging the gap between the constant and variable returns-to-scale models: Selective proportionality in data envelopment analysis. J. Oper. Res. Soc. 55(3):265-276.

Podinovski VV (2004b) Production trade-offs and weight restrictions in data envelopment analysis. J. Oper. Res. Soc. 55(12):1311-1322.

Podinovski VV (2009) Production technologies based on combined proportionality assumptions. J. Productivity Anal. 32(1):21-26. 
Podinovski et al.: Marginal Values for Production Frontiers

Article submitted to Operations Research; manuscript no. (Please, provide the mansucript number!)

Podinovski VV, Bouzdine-Chameeva T (2013) Weight restrictions and free production in data envelopment analysis. Oper. Res. 61(2):426-437.

Podinovski VV, Førsund FR (2010) Differential characteristics of efficient frontiers in data envelopment analysis. Oper. Res. 58(6):1743-1754.

Podinovski VV, Førsund FR, Krivonozhko VE (2009) A simple derivation of scale elasticity in data envelopment analysis. Eur. J. Oper. Res. 197(1):149-153.

Podinovski VV, Ismail I, Bouzdine-Chameeva T, Zhang W (2014) Combining the assumptions of variable and constant returns to scale in the efficiency evaluation of secondary schools. Eur. J. Oper. Res. 239(2):505-513.

Rockafellar RT (1970) Convex Analysis (Princeton University Press, Princeton, NJ).

Roos C, Terlaky T, Vial JP (2005) Interior Point Methods for Linear Optimization (Springer Science + Business Media, New York).

Rosen D, Schaffnit C, Paradi JC (1998) Marginal rates and two-dimensional level curves in DEA. J. Productivity Anal. 9(3):205-232.

Ruggiero J (2000) Nonparametric estimation of returns to scale in the public sector with an application to the provision of educational services. J. Oper. Res. Soc. 51(8):906-912.

Sahoo BK, Zhu J, Tone K, Klemen BM (2014) Decomposing technical efficiency and scale elasticity in two-stage network DEA. Eur. J. Oper. Res. 233(3):584-594.

Seiford LM, Thrall RM (1990) Recent developments in DEA: The mathematical programming approach to frontier analysis. J. Econometrics 46(1-2):7-48.

Shephard RW (1974) Indirect Production Functions. Mathematical Systems in Economics No. 10. (Anton Hain, Meisenheim am Glan).

Thanassoulis E, Portela MCS, Despić O (2008) Data envelopment analysis: The mathematical programming approach to efficiency analysis. Fried HO, Lovell CAK, Schmidt SS, eds. The Measurement of Productive Efficiency and Productivity Growth (Oxford University Press, New York) 251-420.

Tone K (2001) On returns to scale under weight restrictions in data enverlopment analysis. J. Productivity Anal. 16(1):31-47.

Zelenyuk V (2013) A scale elasticity measure for directional distance function and its dual: Theory and DEA estimation. Eur. J. Oper. Res. 228(3):592-600. 